\title{
Advanced optical effective medium modeling for a single layer of polydisperse ellipsoidal nanoparticles embedded in a homogeneous dielectric medium: Surface plasmon resonances
}

\author{
Johann Toudert, ${ }^{1, *}$ Lionel Simonot, ${ }^{2}$ Sophie Camelio, ${ }^{2}$ and David Babonneau ${ }^{2}$ \\ ${ }^{1}$ Instituto de Óptica, Laser Processing Group, CSIC, Serrano 121, 28006 Madrid, Spain \\ ${ }^{2}$ Institut PPRIME, UPR 3346 CNRS, Université de Poitiers, Bât. SP2MI, Boulevard Marie et Pierre Curie, BP 30179, \\ 86962 Futuroscope Chasseneuil Cedex, France
}

(Received 16 December 2011; revised manuscript received 9 March 2012; published 10 July 2012)

\begin{abstract}
We calculate, in the quasistatic coupled dipole approximation, the analytical expressions of the effective dielectric tensor of a single layer of polydisperse ellipsoidal nanoparticles with two of their principal axes in the layer's plane and embedded in a homogeneous dielectric medium. The organization (isotropic or anisotropic) and orientation (without or with a preferential in-plane orientation) of the nanoparticles is taken into account, together with their (possibly correlated) in-plane size, in-plane projected shape, and height distributions. In particular, we propose to describe the response of a layer of nanoparticles presenting a height distribution by using a vertically graded effective medium model. The expressions are tested in the case of finely characterized dielectric/silver/dielectric granular trilayers grown by means of vapor deposition in which the silver coalesced nanoparticles present correlated in-plane size and in-plane projected shape/height distributions and a moderate surface coverage of about $25 \%$. A satisfactory quantitative agreement is obtained between the simulated and measured surface plasmon extinction bands of the metal nanoparticles. This agreement is permitted by the capability of the effective medium model of taking into account the ellipsoidal shape of the nanoparticles. The significant role of the size and shape distributions is also demonstrated.
\end{abstract}

DOI: 10.1103/PhysRevB.86.045415

PACS number(s): 78.20.Bh, 78.67.Bf, 78.68.+m

\section{INTRODUCTION}

Nanoparticles (NPs) are known to present optical properties strongly different from those of the corresponding bulk materials. In particular, the confinement of conduction electrons in metal NPs gives rise to localized surface plasmon resonance (SPR) modes, ${ }^{1}$ which are responsible for a strong absorption of light, ${ }^{1}$ the enhancement of the electromagnetic field around the NPs (near field) ${ }^{2}$ and of the scattering to the far field. ${ }^{1}$ These features make metal NPs interesting candidates for the development of thin films-based all-optical integrated devices. Nanocomposite thin films made of metal NPs supported on a substrate or embedded in a homogeneous dielectric medium can be tailored to present tunable SPR extinction bands in the visible, near-infrared or near-ultraviolet range, ${ }^{3,4}$ a high nonlinear third-order susceptibility, ${ }^{5,6}$ an ultrafast time response, ${ }^{7}$ to allow an enhancement or quenching of the luminescence of nearby emitters ${ }^{8,9}$ or to trap light in photovoltaic media. ${ }^{10}$ It is well known that the optical response of such thin films is strongly affected by their nanostructure (nature, ${ }^{1}$ size, ${ }^{1}$ shape, ${ }^{1,3,4}$ organization, ${ }^{1,11-13}$ dielectric environment of the $\mathrm{NPs}^{1,14}$ ). Designing such materials with an optimized optical response therefore requires addressing the influence of the different structural parameters. For this purpose, plenty of experimental and theoretical works were undertaken, leading to the development of models and methods permitting to link nanostructure and optical response. Such models and methods are also needed for the determination of the nanostructure from noninvasive optical measurements such as spectroscopic ellipsometry, ${ }^{15,16}$ differential reflectance spectroscopy, ${ }^{17,18}$ optical transmittance, ${ }^{11}$ or scattering measurements. ${ }^{19}$ The early calculations of $\mathrm{Mie}^{1,20}$ permitted the determination of the optical response of metal spheres of various sizes in the framework of classical electrodynamics. More recently, classical numerical methods, based on a modal decomposition of the electromagnetic fields or potentials ( $T$ matrix ${ }^{21}$ and multiple multipole ${ }^{22}$ methods) or on volume discretization (discrete dipole approximation, ${ }^{23,24}$ finite element method, ${ }^{25,26}$ finite difference time domain ${ }^{25,26}$ ) or surface discretization (surface integral methods such as the boundary element $\operatorname{method}^{27,28}$, were used to compute accurately the optical response of metal NPs of various shapes, sizes, either isolated or forming dimers or more complex aggregates. Quantum approaches supported the study of the optical extinction of very small NPs (diameter lower than $5 \mathrm{~nm})^{29}$ for which a classical treatment is usually not appropriate, and permitted a more realistic calculation of the near-field enhancement at the surface of $\mathrm{NPs}^{30}$ than classical calculations. These methods are, nevertheless, time and resources consuming, and their use is generally restricted to systems involving a small number of NPs. In the case of larger systems, such as thin films consisting of a statistical assembly of supported or embedded interacting NPs, approximations are usually made. The effect of interactions between radiating NPs arranged in 2D periodic arrays with a low or moderate surface coverage was reasonably described using the coupled dipole approximation,,$^{13}$ where each NP is considered as a point dipole. The point dipole approximation is also at the basis of many effective medium models, which hold when the NPs are much smaller than the wavelength of the light, so that they do not significantly scatter and fulfill quasistatic conditions. In this small-size limit, it is usually claimed that a heterogeneous nanocomposite material can be considered as a homogeneous medium whose macroscopic optical response is described by an effective dielectric tensor. Effective medium theories were developed for a $3 \mathrm{D}^{31-35}$ or a $2 \mathrm{D}$ organization ${ }^{36,37}$ of the NPs. In the frequently encountered case of single layers of NPs (2D organization), the simplest method was 
proposed by Yamaguchi et al. three decades ago. ${ }^{36}$ The NPs were first assumed to be monodisperse, distributed at the nodes of a square lattice, supported on a substrate or embedded in a homogeneous dielectric medium and to present a spheroidal shape (with the revolution axis perpendicular to the NPs layer plane). They were considered as interacting point dipoles and, when supported, the influence of the substrate was taken into account by image dipole effects. This model was shown to fail in describing rigorously the NP-substrate interaction, ${ }^{38,39}$ and the interaction between closely packed $\mathrm{NPs}^{22}$ (high surface coverage), which involve multipolar contributions to the quasistatic electric potential. In addition, it requires defining an optical thickness for the single layer of NPs, whose exact value is linked to the features of the 2D array of NPs. ${ }^{40-42}$ An alternative and more general effective medium approach suitable to the case of nanocomposite thin films, and rubbing out the concept of optical thickness, was proposed by Bedeaux and Vlieger, in the framework of the surface susceptibility method. ${ }^{40-42}$ It permits, for instance, to deal with 2D polydisperse assemblies of NPs distributed homogeneously and isotropically on a substrate or in a homogeneous medium, and presenting correlated sizes, shapes and positions. It was then mainly applied to monodisperse spheroidal or truncated spheroidal supported NPs (with their revolution axis perpendicular to the substrate) whose optical response was calculated using multipolar expansions of the quasistatic electric potential. ${ }^{42-46}$ In contrast, little has been done using the surface susceptibility method regarding polydisperse ellipsoidal NPs, ${ }^{47}$ which are nevertheless frequently encountered in nanocomposite thin films. ${ }^{48,49}$ Indeed, the GRANFILM code, ${ }^{50}$ based on the Bedeaux and Vlieger's approach, only permits calculations for supported NPs with a revolution axis perpendicular to the substrate and ignores the effects of size and shape distributions, together with their possible correlations. Due to its complex parameterization, the implementation of the full polydisperse Bedeaux and Vlieger's model does not seem to be straightforward, thus making the development of more specific models desirable. To our knowledge, little has been reported about easily implementable effective medium models suitable for the case of single layers of polydisperse ellipsoidal NPs, with two of their principal axes being in the layer's plane. ${ }^{51}$ Especially, the influence of a polydisperse height distribution of the NPs, which may be correlated to their in-plane effective size and in-plane projected shape in real systems, has not been examined so far.

In this paper, we thus calculate, in the quasistatic coupled dipole approximation, simple analytical expressions giving the effective optical response of a single layer of NPs embedded in a homogeneous dielectric medium (as it is the case when the NPs are sandwiched between two dielectric layers having the same dielectric function) with as few additional approximations as possible. We will first present the calculations in a general case of ellipsoidal NPs with two of their principal axes in the layer's plane. The height of these NPs will be fixed and the length of their in-plane axes will be considered as polydisperse. No assumption will be made about their in-plane organization and orientation (see Sec. II). The calculation will then be derived to match the specific case of polydisperse NPs with a random in-plane orientation and an isotropic in-plane organization, together with a fixed height and correlated in-plane size and in-plane projected shape distributions (see Sec. III). It will then be extended to NPs with a polydisperse height distribution-correlated to the in-plane polydispersity-the single layer of NPs being tentatively described in that case by a vertically graded effective medium (see Sec. IV). The influence of the different structural parameters on the SPRs of metal NPs will then be studied from simulations (see Sec. V). Vapor-deposited dielectric/Ag/dielectric granular trilayered films, in which the previous conditions are satisfied (polydispersity with in-plane size/in-plane projected shape/height correlation, random in-plane orientation and isotropic in-plane organization of the NPs) and with a moderate surface coverage of about $25 \%$, will finally be used as model materials to test the theory (see Sec. VI). Transmission electron microscopy (TEM) on these films provides accurate input parameters for the calculations, the results of which will be compared to experimental optical transmittance data, displaying marked SPRs sensitive to the NPs sizes and shapes.

\section{GENERAL CALCULATION: ELLIPSOIDAL NPS WITH IN-PLANE POLYDISPERSITY AND CONSTANT HEIGHT}

\section{A. Hypothesis and method}

Effective medium modeling consists in considering a heterogeneous material as a homogeneous medium presenting identical macroscopic physical properties. In the case of a single layer of NPs with a constant height $H$ embedded in a homogeneous dielectric medium, the common sense leads to consider that the effective medium is a homogeneous thin layer of (optical) thickness $t$. For monodisperse spherical NPs, we showed theoretically that it is legitimate to take $t=H$ for calculating usual measurable quantities such as optical transmittance or absorbance ${ }^{52}$ at normal incidence, provided the effective medium model is adequately chosen. In an analogous way, we will assume in the following that the thickness of the effective medium layer representing a single layer of polydisperse NPs with a constant height $H$ can be taken equal to $H$, as depicted in Fig. 1(a).
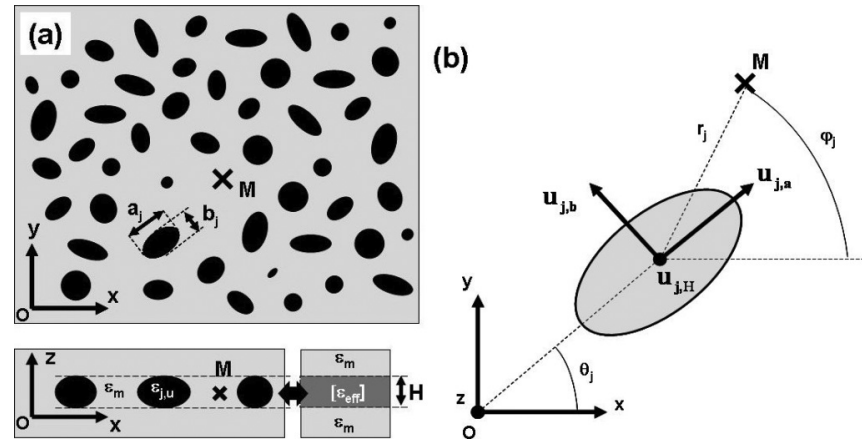

FIG. 1. (a) Schematic representation of a 2D assembly of NPs embedded in a homogeneous dielectric medium, in top view and cross-section view. $M$ is the point at which the local electric field has to be calculated. The in-plane axes $a_{j}$ and $b_{j}$ of a given $j$ th particle are shown. All the NPs are supposed to present the same height $H$, which is also the thickness chosen for the effective medium layer. (b) Top view of the $j$ th NP and orientation of its set of axes. 
The macroscopic optical response of this homogeneous layer is linked to the nanostructure of the single layer of NPs by the effective dielectric tensor $\left[\varepsilon_{\text {eff }}\right]$, whose expression has to be determined. When the NPs are dispersed in a homogeneous dielectric medium whose dielectric function is $\varepsilon_{m}$, the following relation links the macroscopic electric field $\mathbf{E}$ to the macroscopic polarization $\mathbf{P}$ of the nanocomposite material:

$$
\mathbf{P}=\varepsilon_{0}\left(\left[\varepsilon_{\mathrm{eff}}\right]-\varepsilon_{m}\right) \mathbf{E} .
$$

$\mathbf{P}$ is a consequence of polarization events at the nanometer scale, dominated by the polarization of the NPs. Each of them is assumed to fulfill the quasistatic conditions and to be excited by an electric field homogeneous over its whole volume, and will thus be considered as the superposition of three point dipoles (one oriented along each of its axes) located at its mass center. This point dipole approximation is correct for describing the far-field response of a small isolated ellipsoidal NP. ${ }^{53}$ Nevertheless, it fails for deriving properly the electric field at a short distance from the NP ${ }^{38}$ We assume that the interparticle distances involved in this paper are large enough so that the point dipole picture is sufficient for describing the interactions between NPs. In the general case of polydisperse systems, the NPs can be sorted into classes $k$ of identical entities (same size, shape and orientation) of given dipolar momentum $\mathbf{p}_{k}$; one can thus write

$$
\mathbf{P}=\Sigma N_{k} \mathbf{p}_{k} .
$$

In this equation, $N_{k}$ is the number of NPs of the class $k$ per volume unit. The dipolar momentum $\mathbf{p}_{k}$ of a given NP is linked to its polarizability tensor per surface unit $\left[\alpha_{k}\right]$ (named hereafter polarizability tensor for the sake of clarity) and to the local electric field $\mathbf{E}_{\mathrm{loc}, k}$ applied on the NP, using the following relation in which $V_{k}$ is the volume of the NP:

$$
\mathbf{p}_{k}=\varepsilon_{0} \varepsilon_{m} \mathrm{~V}_{k}\left[\alpha_{k}\right] \mathbf{E}_{\mathrm{loc}, k}
$$

As it can be seen from Eqs. (1)-(3), the calculation of the local electric field applied on each NP is mandatory for determining the macroscopic polarization and $\left[\varepsilon_{\text {eff }}\right]$. The calculation of $\left[\varepsilon_{\text {eff }}\right]$ will therefore be done by the following method. First, the local electric field created at an arbitrary NP will be calculated by taking into account the contribution of the surrounding ones. Since the NPs are assumed to be embedded in a homogeneous dielectric medium, no contribution from image dipoles has to be taken into account in the calculation, in contrast with the case of NPs supported on a substrate. Then, assuming that the NPs present a homogeneous in-plane organization, i.e., that each NP experiences the same local electric field $\mathbf{E}_{\mathrm{loc}, k}=\mathbf{E}_{\mathrm{loc}}$, the macroscopic polarization will be derived as well as $\left[\varepsilon_{\text {eff }}\right]$.

\section{B. Calculations}

\section{Local field created at an arbitrary NP}

In order to calculate the local field $\mathbf{E}_{\text {loc }}$ created at a given point $\mathrm{M}$ by the $2 \mathrm{D}$ assembly of NPs, whose topology is presented schematically in Fig. 1(a), it is first necessary to determine the field created by one of the NPs. Figure 1(b) shows one of them ( $j$ th NP), whose in-plane position relative to $\mathrm{M}$ is defined by $r_{j}$ and $\varphi_{j}$. In the $(x, y, z)$ set of axes, the dipolar momentum of this NP can be written as $\mathbf{p}_{\mathbf{j}}=\left(p_{j, x}, p_{j, y}, p_{j, z}\right)$. Its nonretarded contribution to the local field at $\mathrm{M}$ can be written in a matrix form:

$$
\mathbf{E}_{\mathbf{i n t} / p_{j}}=\frac{1}{4 \pi \varepsilon_{0} \varepsilon_{m} r_{j}{ }^{3}}\left[\begin{array}{ccc}
3 \cos ^{2} \varphi_{j}-1 & 3 \cos \varphi_{j} \sin \varphi_{j} & 0 \\
3 \cos \varphi_{j} \sin \varphi_{j} & 3 \sin ^{2} \varphi_{j}-1 & 0 \\
0 & 0 & -1
\end{array}\right]\left(\begin{array}{l}
p_{j, x} \\
p_{j, y} \\
p_{j, z}
\end{array}\right)
$$

By summing the contributions of all the NPs and taking into account the external electric field $\mathbf{E}_{\mathbf{0}}$, which is linked to the macroscopic field $\mathbf{E}$ inside the effective layer by the boundary relation $\left(E_{0 x}, E_{0 y}, E_{0 z}\right)=\left(E_{x}, E_{y}, \varepsilon_{m}^{-1} \varepsilon_{z z} E_{z}\right)$, one obtains $\mathbf{E}_{\mathrm{loc}}=\left(E_{\mathrm{loc}, x}, E_{\mathrm{loc}, y}, E_{\mathrm{loc}, z}\right)$ :

$$
\left(\begin{array}{c}
E_{\mathrm{loc}, x} \\
E_{\mathrm{loc}, y} \\
E_{\mathrm{loc}, z}
\end{array}\right)=\left(\begin{array}{c}
E_{x} \\
E_{y} \\
\varepsilon_{m}^{-1} \varepsilon_{z z} E_{z}
\end{array}\right)+\frac{1}{4 \pi \varepsilon_{0} \varepsilon_{m}} \sum_{j}\left(\frac{1}{r_{j}{ }^{3}}\left[\begin{array}{ccc}
3 \cos ^{2} \varphi_{j}-1 & 3 \cos \varphi_{j} \sin \varphi_{j} & 0 \\
3 \cos \varphi_{j} \sin \varphi_{j} & 3 \sin ^{2} \varphi_{j}-1 & 0 \\
0 & 0 & -1
\end{array}\right]\left(\begin{array}{c}
p_{j, x} \\
p_{j, y} \\
p_{j, z}
\end{array}\right)\right) .
$$

In this relation, $\varepsilon_{z z}$ is the vertical component of the effective dielectric tensor [ $\left.\varepsilon_{\mathrm{eff}}\right]$. In order to further derive $\mathbf{E}_{\mathrm{loc}}$ from Eq. (5), it is necessary to determine the components of the $\mathbf{p}_{j}$ 's in the $(x, y, z)$ system of axes. The $\mathbf{p}_{j}$ 's verify the relation $\mathbf{p}_{j}=\varepsilon_{0} \varepsilon_{m} V_{j}\left[\alpha_{j}\right] \mathbf{E}_{\text {loc }}$ [see Eq. (3)], the components of $\left[\alpha_{j}\right]$ being well known in the $\left(\mathbf{u}_{j, a}, \mathbf{u}_{j, b}, \mathbf{u}_{j, H}\right)$ set of axes attached to the NP: $:^{53}$

$$
\left[\alpha_{j}\right]=\left(\begin{array}{ccc}
\alpha_{j, a} & 0 & 0 \\
0 & \alpha_{j, b} & 0 \\
0 & 0 & \alpha_{j, H}
\end{array}\right)
$$

Since the ( $\left.\mathbf{u}_{j, a}, \mathbf{u}_{j, b}, \mathbf{u}_{j, H}\right)$ set of axes is simply tilted by an angle $\theta_{j}$ in the horizontal plane with respect to the ( $\left.x, y, z\right)$ set of axes, the $\mathbf{p}_{j}$ 's are obtained by introducing the rotation and inverse rotation matrices corresponding to a rotation angle $\theta_{j}$. One 
thus obtains

$$
\mathbf{p}_{j}=\left(\begin{array}{l}
p_{j, x} \\
p_{j, y} \\
p_{j, z}
\end{array}\right)=\varepsilon_{0} \varepsilon_{m} V_{j}\left(\begin{array}{lll}
\cos \theta_{j} & -\sin \theta_{j} & 0 \\
\sin \theta_{j} & \cos \theta_{j} & 0 \\
0 & 0 & 1
\end{array}\right)\left(\begin{array}{ccc}
\alpha_{j, a} & 0 & 0 \\
0 & \alpha_{j, b} & 0 \\
0 & 0 & \alpha_{j, H}
\end{array}\right)\left(\begin{array}{lll}
\cos \theta_{j} & \sin \theta_{j} & 0 \\
-\sin \theta_{j} & \cos \theta_{j} & 0 \\
0 & 0 & 1
\end{array}\right)\left(\begin{array}{l}
E_{\mathrm{loc}, x} \\
E_{\mathrm{loc}, y} \\
E_{\mathrm{loc}, z}
\end{array}\right) .
$$

The components of $\mathbf{p}_{j}$ can then be written

$$
\mathbf{p}_{j}=\left(\begin{array}{l}
p_{j, x} \\
p_{j, y} \\
p_{j, z}
\end{array}\right)=\varepsilon_{0} \varepsilon_{m} V_{j}\left(\begin{array}{ccc}
A_{j, x x} & A_{j, x y} & 0 \\
A_{j, y x} & A_{j, y y} & 0 \\
0 & 0 & A_{j, z z}
\end{array}\right)\left(\begin{array}{l}
E_{\mathrm{loc}, x} \\
E_{\mathrm{loc}, y} \\
E_{\mathrm{loc}, z}
\end{array}\right) \quad \text { with } \quad \begin{aligned}
& A_{j, x x}=\alpha_{j, a} \cos ^{2} \theta_{j}+\alpha_{j, b} \sin ^{2} \theta_{j} \\
& A_{j, x y}=\left(\alpha_{j, a}-\alpha_{j, b}\right) \cos \theta_{j} \sin \theta_{j} \\
& A_{j, y x}=\left(\alpha_{j, a}-\alpha_{j, b}\right) \cos \theta_{j} \sin \theta_{j} \\
& A_{j, y y}=\alpha_{j, a} \sin ^{2} \theta_{j}+\alpha_{j, b} \cos ^{2} \theta_{j} \\
& A_{j, z z}=\alpha_{j, H} .
\end{aligned}
$$

The $A_{j, u v}$ 's carry the information about the $j$ th NP's in-plane orientation via the $\theta_{j}$ 's and about its shape and size, which govern the components of its polarizability tensor through ${ }^{53}$

$$
\alpha_{j, w}=\frac{\varepsilon_{j, w}-\varepsilon_{m}}{\varepsilon_{m}+L_{j, w}\left(\varepsilon_{j, w}-\varepsilon_{m}\right)}, \quad \text { with } w=a, b \text {, or } H .
$$

$\varepsilon_{j, w}$ are the (possibly size-dependent) dielectric functions of the NP along the axis $w$ and the $L_{j, w}$ 's are the depolarization factors of the ellipsoidal NP, which are linked to its shape via the following relation: ${ }^{53}$

$$
L_{j, w}=\int_{0}^{\infty} \frac{a_{j} b_{j} H_{j} d X}{16\left(X+w_{j}^{2} / 4\right) \sqrt{\left(X+{\frac{a_{j}}{4}}^{2}\right)\left(X+{\frac{b_{j}}{4}}^{2}\right)\left(X+{\frac{H_{j}}{4}}^{2}\right)}},
$$

in which $a_{j}$ and $b_{j}$ are the lengths of the in-plane long and short axis of the $j$ th NP, respectively, and $H_{j}(=H)$ its height, as depicted in Fig. 1(a). Now introducing the components of the $\mathbf{p}_{j}$ 's into Eq. (5) and after regrouping the terms, one gets

$$
\begin{aligned}
&\left(\begin{array}{l}
E_{\mathrm{loc}, x} \\
E_{\mathrm{loc}, y} \\
E_{\mathrm{loc}, z}
\end{array}\right)=\left(\begin{array}{l}
E_{x} \\
E_{y} \\
\varepsilon_{m}^{-1} \varepsilon_{z z} E_{z}
\end{array}\right)+\left(\begin{array}{ccc}
B_{x x} & B_{x y} & 0 \\
B_{y x} & B_{y y} & 0 \\
0 & 0 & B_{z z}
\end{array}\right)\left(\begin{array}{l}
E_{\mathrm{loc}, x} \\
E_{\mathrm{loc}, y} \\
E_{\mathrm{loc}, z}
\end{array}\right) \\
& \text { with }\left\{\begin{array}{l}
B_{x x}=\frac{1}{4 \pi}\left\{\sum_{j}\left[\frac{V_{j}}{r_{j}{ }^{3}}\left[\left(3 \cos ^{2} \varphi_{j}-1\right) A_{j, x x}+3 \cos \varphi_{j} \sin \varphi_{j} A_{j, y x}\right]\right]\right\} \\
B_{x y}=\frac{1}{4 \pi}\left\{\sum_{j}\left[\frac{V_{j}}{r_{j}{ }^{3}}\left[\left(3 \cos ^{2} \varphi_{j}-1\right) A_{j, x y}+3 \cos \varphi_{j} \sin \varphi_{j} A_{j, y y}\right]\right]\right\} \\
B_{y x}=\frac{1}{4 \pi}\left\{\sum_{j}\left[\frac{V_{j}}{r_{j}{ }^{3}}\left[3 \cos \varphi_{j} \sin \varphi_{j} A_{j, x x}+\left(3 \sin ^{2} \varphi_{j}-1\right) A_{j, y x}\right]\right]\right\} . \\
B_{y y}=\frac{1}{4 \pi}\left\{\sum_{j}\left[\frac{V_{j}}{r_{j}{ }^{3}}\left[3 \cos \varphi_{j} \sin \varphi_{j} A_{j, x y}+\left(3 \sin ^{2} \varphi_{j}-1\right) A_{j, y y}\right]\right]\right\} \\
B_{z z}=\frac{1}{4 \pi}\left\{\sum_{j}\left[\frac{-V_{j} A_{j, z z}}{r_{j}{ }^{3}}\right]\right\}
\end{array}\right.
\end{aligned}
$$

Equation (11) forms a linear system whose solutions are the components of the local electric field $\mathbf{E}_{\mathrm{loc}}$ at an arbitrary point $\mathbf{M}$, as a function of the macroscopic electric field $\mathbf{E}$ and the topology of the surrounding material (given by the $B_{u v}$ 's, that gather all the information about the NPs shape, size and orientation - through the $A_{j, u v}$ 's — and about their organization through the $r_{j}$ 's and $\varphi_{j}$ 's):

$$
\left(\begin{array}{l}
E_{\mathrm{loc}, x} \\
E_{\mathrm{loc}, y} \\
E_{\mathrm{loc}, z}
\end{array}\right)=\left(\begin{array}{lcc}
1-B_{x x} & B_{x y} & 0 \\
B_{y x} & 1-B_{y y} & 0 \\
0 & 0 & 1-B_{z z}
\end{array}\right)^{-1}\left(\begin{array}{l}
E_{x} \\
E_{y} \\
\varepsilon_{m}^{-1} \varepsilon_{z z} E_{z}
\end{array}\right)
$$

\section{Dipolar momentum of one particle excited by the local electric field}

It comes from Eq. (12) that the local field $\mathbf{E}_{\text {loc }}$ can be written as

$$
\left(\begin{array}{l}
E_{\mathrm{loc}, x} \\
E_{\mathrm{loc}, y} \\
E_{\mathrm{loc}, z}
\end{array}\right)=\left(\begin{array}{ccc}
C_{x x} & C_{x y} & 0 \\
C_{y x} & C_{y y} & 0 \\
0 & 0 & C_{z z}
\end{array}\right)\left(\begin{array}{l}
E_{x} \\
E_{y} \\
\varepsilon_{m}^{-1} \varepsilon_{z z} E_{z}
\end{array}\right) \quad \text { with } \quad\left\{\begin{array}{l}
C_{x x}=\frac{\left(1-B_{y y}\right)}{\left(1-B_{y y}\right)\left(1-B_{x x}\right)-B_{x y} B_{y x}} \\
C_{x y}=\frac{B_{x y}}{\left(1-B_{y y}\right)\left(1-B_{x x}\right)-B_{x y} B_{y x}} \\
C_{y x}=\frac{B_{y x}}{\left(1-B_{y y}\right)\left(1-B_{x x}\right)-B_{x y} B_{y x}} \\
C_{y y}=\frac{\left(1-B_{x x}\right)}{\left(1-B_{y y}\right)\left(1-B_{x x}\right)-B_{x y} B_{y x}} \\
C_{z z}=\frac{1}{\left(1-B_{z z}\right)}
\end{array} .\right.
$$


After combining Eqs. (8) and (13), one obtains the dipolar momentum $\mathbf{p}_{k}$ of a given $k$ th NP excited by $\mathbf{E}_{\text {loc }}$ :

$$
\mathbf{p}_{k}=\left(\begin{array}{l}
p_{k, x} \\
p_{k, y} \\
p_{k, z}
\end{array}\right)=\varepsilon_{0} \varepsilon_{m} V_{k}\left(\begin{array}{ccc}
A_{k, x x} & A_{k, x y} & 0 \\
A_{k, y x} & A_{k, y y} & 0 \\
0 & 0 & A_{k, z z}
\end{array}\right)\left(\begin{array}{ccc}
C_{x x} & C_{x y} & 0 \\
C_{y x} & C_{y y} & 0 \\
0 & 0 & C_{z z}
\end{array}\right)\left(\begin{array}{l}
E_{x} \\
E_{y} \\
\varepsilon_{m}^{-1} \varepsilon_{z z} E_{z}
\end{array}\right) .
$$

After calculating the matrix products, Eq. (14) gives $\mathbf{p}_{k}$ as a function of the $k$ th NP's size (through $V_{k}$ and the $A_{k, u v}$ 's), shape and orientation (through the $A_{k, u v}$ 's), the topology of the surrounding material (through the $C_{u v}$ 's) and the macroscopic electric field $\mathbf{E}$ :

$$
\mathbf{p}_{k}=\left(\begin{array}{l}
p_{k, x} \\
p_{k, y} \\
p_{k, z}
\end{array}\right)=\varepsilon_{0} \varepsilon_{m} V_{k}\left(\begin{array}{ccc}
F_{k, x x} & F_{k, x y} & 0 \\
F_{k, y x} & F_{k, y y} & 0 \\
0 & 0 & F_{k, z z}
\end{array}\right)\left(\begin{array}{l}
E_{x} \\
E_{y} \\
\varepsilon_{m}^{-1} \varepsilon_{z z} E_{z}
\end{array}\right) \text { with }\left\{\begin{array}{l}
F_{k, x x}=A_{k, x x} C_{x x}+A_{k, x y} C_{y x} \\
F_{k, x y}=A_{k, x x} C_{x y}+A_{k, x y} C_{y y} \\
F_{k, y x}=A_{k, y x} C_{x x}+A_{k, y y} C_{y x} . \\
F_{k, y y}=A_{k, y x} C_{x y}+A_{k, y y} C_{y y} \\
F_{k, z z}=A_{k, z z} C_{z z}
\end{array}\right.
$$

\section{Macroscopic polarization and effective dielectric tensor}

On one hand, from Eqs. (2) and (15), one obtains the macroscopic polarization $\mathbf{P}$ as a function of the $F_{k, u v}$ factors, which are linked to the size, shape, orientation, and organization of the NPs:

$$
\mathbf{P}=\sum_{k}\left[N_{k} \varepsilon_{0} \varepsilon_{m} V_{k}\left(\begin{array}{ccc}
F_{k, x x} & F_{k, x y} & 0 \\
F_{k, y x} & F_{k, y y} & 0 \\
0 & 0 & \varepsilon_{m}^{-1} \varepsilon_{z z} F_{k, z z}
\end{array}\right)\left(\begin{array}{c}
E_{x} \\
E_{y} \\
E_{z}
\end{array}\right)\right] .
$$

On the other hand, from Eq. (1), the macroscopic polarization is linked to the effective dielectric function of the nanocomposite material:

$$
\mathbf{P}=\varepsilon_{0}\left(\begin{array}{ccc}
\varepsilon_{x x}-\varepsilon_{m} & \varepsilon_{x y} & \varepsilon_{x z} \\
\varepsilon_{y x} & \varepsilon_{y y}-\varepsilon_{m} & \varepsilon_{y z} \\
\varepsilon_{z x} & \varepsilon_{z y} & \varepsilon_{z z}-\varepsilon_{m}
\end{array}\right)\left(\begin{array}{c}
E_{x} \\
E_{y} \\
E_{z}
\end{array}\right) .
$$

After combining Eqs. (16) and (17), one obtains the final expression of the effective dielectric tensor of the nanocomposite material consisting of a single layer of NPs of constant height $\mathrm{H}$ and embedded in a homogeneous dielectric medium with a dielectric function $\varepsilon_{m}$ :

$$
\left[\varepsilon_{\text {eff }}\right]=\left(\begin{array}{ccc}
\varepsilon_{x x} & \varepsilon_{x y} & 0 \\
\varepsilon_{y x} & \varepsilon_{y y} & 0 \\
0 & 0 & \varepsilon_{z z}
\end{array}\right)=\left(\begin{array}{ccc}
\varepsilon_{m}\left[1+\sum_{k} N_{k} V_{k} F_{k, x x}\right] & \varepsilon_{m}\left[\sum_{k} N_{k} V_{k} F_{k, x y}\right] & 0 \\
\varepsilon_{m}\left[\sum_{k} N_{k} V_{k} F_{k, y x}\right] & \varepsilon_{m}\left[1+\sum_{k} N_{k} V_{k} F_{k, y y}\right] & 0 \\
0 & 0 & \varepsilon_{m}\left[1-\sum_{k} N_{k} V_{k} F_{k, z z}\right]^{-1}
\end{array}\right)
$$

Due to the 2D organization of the NPs, it can be useful to introduce the surface densities $N_{s, k}$ of NPs in Eq. (18). Since the thickness of the effective medium layer is $\mathrm{H}$, one can write $N_{k}=N_{s, k} / H$. Since no restrictive hypothesis was made on the in-plane organization and orientation of the NPs, the obtained formulas are suitable to the case of NPs with a random or preferential in-plane orientation, distributed isotropically or at the nodes of an anisotropic array, provided the relation $\mathbf{E}_{\mathrm{loc}, k}=\mathbf{E}_{\mathrm{loc}}$ holds. In addition, the summation on $k$ permits to take in-plane size and in-plane projected shape distributions into account. In the following section, we apply Eq. (18) to the case of a polydisperse assembly of ellipsoidal NPs embedded in a homogeneous dielectric medium with a random in-plane orientation and an isotropic in-plane organization.

\section{APPLICATION: RANDOM IN-PLANE ORIENTATION AND ISOTROPIC IN-PLANE ORGANIZATION OF NPS WITH CONSTANT HEIGHT}

\section{A. Polydisperse case: NPs with in-plane size/in-plane projected shape correlation}

Embedded NPs grown by vapor deposition techniques usually display an isotropic in-plane organization, a random in-plane orientation and present correlated distributions of their in-plane size and in-plane projected shape. ${ }^{48,49}$ The general equations obtained in Sec. II (sets of coefficients $A_{j, u v}$, $B_{u v}, F_{k, u v}$ ) can be adapted to obtain simple formulas that take into account these features. The in-plane size of the $j$ th NP is given by its in-plane effective diameter $D_{j}=\left(a_{j} b_{j}\right)^{1 / 2}$, and 
its in-plane projected shape depends on the values of the axes lengths $a_{j}$ and $b_{j}$, the values of which are assumed to be correlated to $D_{j}$, following the relations $a_{j}=a\left(D_{j}\right)$ and $b_{j}=$ $b\left(D_{j}\right)$, usually written under the form $b_{j} / a_{j}=b / a\left(D_{j}\right)$. The polarizabilities therefore depend on $D_{j}$ and $H$. Accordingly, the $A_{j, u v}$ coefficients of the $j$ th NP only depend on $D_{j}, H$, and its in-plane orientation (given by $\theta_{j}$ ). One thus obtains from Eq. (8),

$$
\begin{aligned}
& A_{x x}\left(D_{j}, H, \theta_{j}\right)=\alpha_{a}\left(D_{j}, H\right) \cos ^{2} \theta_{j}+\alpha_{b}\left(D_{j}, H\right) \sin ^{2} \theta_{j} \\
& A_{x y}\left(D_{j}, H, \theta_{j}\right)=\left[\alpha_{a}\left(D_{j}, H\right)-\alpha_{b}\left(D_{j}, H\right)\right] \cos \theta_{j} \sin \theta_{j} \\
& A_{y x}\left(D_{j}, H, \theta_{j}\right)=\left[\alpha_{a}\left(D_{j}, H\right)-\alpha_{b}\left(D_{j}, H\right)\right] \cos \theta_{j} \sin \theta_{j} \\
& A_{y y}\left(D_{j}, H, \theta_{j}\right)=\alpha_{a}\left(D_{j}, H\right) \sin ^{2} \theta_{j}+\alpha_{b}\left(D_{j}, H\right) \cos ^{2} \theta_{j} \\
& A_{z z}\left(D_{j}, H, \theta_{j}\right)=\alpha_{H}\left(D_{j}, H\right)
\end{aligned}
$$

In order to obtain the expression of the $B_{u v}$ coefficients from Eq. (11), one can assume that the discrete sums over the NPs $j$ can be replaced by integrals over a 4D space with NPs position (given by $\varphi$ and $r$ ), orientation (given by $\theta$ ), and size (given by $D$ ) as coordinates. Knowing the surface density $N_{s}(r, \varphi, \theta, D)$ of NPs whose coordinates in this 4D space are $r, \varphi, \theta$, and $D$, one obtains the number $d^{4} N_{\mathrm{s}}(r, \varphi, D, \theta)$ of NPs with an in-plane effective diameter between $D$ and $D+d D$, an orientation angle between $\theta$ and $\theta+d \theta$, which are located inside a small area $d S=r d \varphi d r$ of the $(x, y)$ plane:

$$
d^{4} N_{s}(r, \varphi, D, \theta)=N_{s}(r, \varphi, D, \theta) r d \varphi d r d D d \theta .
$$

In the case of a random in-plane orientation and an isotropic organization of the NPs, one can write

$$
N_{s}(r, \varphi, D, \theta)=N_{s}(r) G(D) / 2 \pi,
$$

where $G(D)$ is the normalized size distribution of the NPs and $N_{s}(r)$ is the number of NPs per unit area located at the distance $r$ from the central point M, i.e., the pair correlation function. Equation (11) can thus be written as

$B_{x x}=\frac{1}{4 \pi} \int_{r=0}^{\infty} \int_{\varphi=0}^{2 \pi} \int_{D=0}^{\infty} \int_{\theta=0}^{2 \pi}\left[\frac{V(D, H)}{r^{3}}\left[\left(3 \cos ^{2} \varphi-1\right) A_{x x}(D, H, \theta)+3 \cos \varphi \sin \varphi A_{y x}(D, H, \theta)\right]\right] N_{S}(r) \frac{1}{2 \pi} G(D) r d \varphi d r d D d \theta$,

$B_{x y}=\frac{1}{4 \pi} \int_{r=0}^{\infty} \int_{\varphi=0}^{2 \pi} \int_{D=0}^{\infty} \int_{\theta=0}^{2 \pi}\left[\frac{V(D, H)}{r^{3}}\left[\left(3 \cos ^{2} \varphi-1\right) A_{x y}(D, H, \theta)+3 \cos \varphi \sin \varphi A_{y y}(D, H, \theta)\right]\right] N_{S}(r) \frac{1}{2 \pi} G(D) r d \varphi d r d D d \theta$,

$B_{y x}=\frac{1}{4 \pi} \int_{r=0}^{\infty} \int_{\varphi=0}^{2 \pi} \int_{D=0}^{\infty} \int_{\theta=0}^{2 \pi}\left[\frac{V(D, H)}{r^{3}}\left[3 \cos \varphi \sin \varphi A_{x x}(D, H, \theta)+\left(3 \cos ^{2} \varphi-1\right) A_{y x}(D, H, \theta)\right]\right] N_{S}(r) \frac{1}{2 \pi} G(D) r d \varphi d r d D d \theta$,

$B_{y y}=\frac{1}{4 \pi} \int_{r=0}^{\infty} \int_{\varphi=0}^{2 \pi} \int_{D=0}^{\infty} \int_{\theta=0}^{2 \pi}\left[\frac{V(D, H)}{r^{3}}\left[3 \cos \varphi \sin \varphi A_{x y}(D, H, \theta)+\left(3 \sin ^{2} \varphi-1\right) A_{y y}(D, H, \theta)\right]\right] N_{S}(r) \frac{1}{2 \pi} G(D) r d \varphi d r d D d \theta$,

$B_{z z}=\frac{1}{4 \pi} \int_{r=0}^{\infty} \int_{\varphi=0}^{2 \pi} \int_{D=0}^{\infty} \int_{\theta=0}^{2 \pi}\left[\frac{-V(D, H)}{r^{3}} A_{z z}(D, H)\right] N_{S}(r) \frac{1}{2 \pi} G(D) r d \varphi d r d D d \theta$,

with the volume $V$ of each NP being a function of $D$ and $H$. After inserting the $A_{u v}$ 's taken from Eq. (19) into Eq. (22), separating the integrals and calculating the angular integrals (over $\theta$ and $\varphi$ ), one gets

$$
\begin{aligned}
& B_{x x}=B_{y y}=\frac{1}{8}\left[\int_{r=0}^{\infty} N_{S}(r) \frac{d r}{r^{2}}\right]\left[\int_{D=0}^{\infty}\left(\alpha_{a}(D, H)+\alpha_{b}(D, H)\right) G(D) V(D, H) d D\right] \\
& B_{y x}=B_{x y}=0 \\
& B_{z z}=-\frac{1}{2}\left[\int_{r=0}^{\infty} N_{S}(r) \frac{d r}{r^{2}}\right]\left[\int_{D=0}^{\infty} \alpha_{H}(D, H) V(D, H) G(D) d D\right] .
\end{aligned}
$$

The $C_{u v}$ 's are then obtained from Eq. (13):

$$
\begin{aligned}
C_{x x} & =C_{y y}=\frac{1}{\left(1-B_{x x}\right)}=\frac{1}{\left(1-B_{y y}\right)}=C_{\|} \\
C_{x y} & =C_{y x}=0 \\
C_{z z} & =\frac{1}{\left(1-B_{z z}\right)}=C_{\perp} .
\end{aligned}
$$

The $F_{u v}$ 's of the central NP (with an effective in-plane diameter $D$ and an in-plane orientation described by $\theta$ ) can then be calculated following Eq. (15):

$$
\begin{aligned}
& F_{x x}(D, H, \theta)=C_{\|}\left[\alpha_{a}(D, H) \cos ^{2} \theta+\alpha_{b}(D, H) \sin ^{2} \theta\right] \\
& F_{x y}(D, H, \theta)=F_{y x}(D, H, \theta)=C_{\|} \cos \theta \sin \theta\left[\alpha_{a}(D, H)-\alpha_{b}(D, H)\right] \\
& F_{y y}(D, H, \theta)=C_{\|}\left[\alpha_{a}(D, H) \sin ^{2} \theta+\alpha_{b}(D, H) \cos ^{2} \theta\right] \\
& F_{z z}(D, H, \theta)=C_{\perp} \alpha_{H}(D, H) .
\end{aligned}
$$


The final step of the calculation consists in deriving the components of the dielectric tensor using Eqs. (18) and (25). Since the $F_{u v}$ 's only depend on $H, D$, and $\theta$, the discrete sums over the NPs can be replaced by integrals over the two latter variables. The number $N_{k}$ of identical NPs per volume unit is thus the number of NPs with a given value of $D$ and $\theta$ per unit of volume. Introducing the total surface density $N_{s}$ of NPs (integrated over all the sizes and orientations) and the thickness of the effective medium layer being assumed to be $\mathrm{H}$ (as explained in Sec. II), $N_{k}$ can be replaced by

$$
N(D, \theta)=N_{s} G(D) /(2 \pi H) .
$$

One then obtains from Eqs. (18), (25), and (26) the components of the dielectric tensor:

$$
\begin{aligned}
& \varepsilon_{x x}=\varepsilon_{y y}=\varepsilon_{m}\left[1+\frac{N_{S} C_{\|}}{2 \pi H} \int_{\theta=0}^{2 \pi} \int_{D=0}^{\infty} G(D) V(D, H)\left[\alpha_{a}(D, H) \sin ^{2} \theta+\alpha_{b}(D, H) \cos ^{2} \theta\right] d D d \theta\right] \\
& \varepsilon_{x y}=\varepsilon_{y x}=\varepsilon_{m}\left[\frac{N_{S} C_{\|}}{2 \pi H} \int_{\theta=0}^{2 \pi} \int_{D=0}^{\infty} G(D) V(D, H) \cos \theta \sin \theta\left[\alpha_{a}(D, H)-\alpha_{b}(D, H)\right] d D d \theta\right] \\
& \varepsilon_{z z}=\varepsilon_{m}\left[1-\frac{N_{S} C_{\perp}}{2 \pi H} \int_{\theta=0}^{2 \pi} \int_{D=0}^{\infty} G(D) \alpha_{H}(D, H) V(D, H) d D d \theta\right]^{-1} .
\end{aligned}
$$

In these expressions, the $C$ factors account for interactions in the layer of NPs, whereas the integrals take into account their in-plane size and in-plane projected shape distributions. After replacing these factors, using Eqs. (23) and (24) and integrating over $\theta$, the final expressions of the dielectric functions can be obtained:

$$
\begin{aligned}
& \varepsilon_{x x}=\varepsilon_{y y}=\varepsilon_{m}\left[1+\frac{\frac{N_{S}}{H} \int_{D=0}^{\infty} G(D) V(D, H)\left[\alpha_{a}(D, H)+\alpha_{b}(D, H)\right] d D}{2\left(1-\frac{1}{8}\left[\int_{r=0}^{\infty} N_{S}(r) \frac{d r}{r^{2}}\right]\left\{\int_{D=0}^{\infty} G(D)\left[\alpha_{a}(D, H)+\alpha_{b}(D, H)\right] V(D, H) d D\right\}\right)}\right]=\varepsilon_{\|} \\
& \varepsilon_{x y}=\varepsilon_{y x}=0 \\
& \varepsilon_{z z}=\varepsilon_{m}\left[1-\frac{\frac{N_{S}}{H} \int_{D=0}^{\infty} G(D) \alpha_{H}(D, H) V(D, H) d D}{1+\frac{1}{2}\left[\int_{r=0}^{\infty} N_{S}(r) \frac{d r}{r^{2}}\right]\left[\int_{D=0}^{\infty} G(D) \alpha_{H}(D, H) V(D, H) d D\right]}\right]^{-1}=\varepsilon_{\perp} .
\end{aligned}
$$

\section{B. Monodisperse case}

Equation (27) can be simplified in order to obtain the dielectric functions for identical ellipsoidal NPs embedded in a homogeneous dielectric medium with an isotropic in-plane organization and random in-plane orientation. This time, the polarizabilities can be expressed directly as a function of $a, b$, and $H$ :

$$
\begin{aligned}
\varepsilon_{y y} & =\varepsilon_{x x}=\varepsilon_{m}\left\{1+\frac{N_{S} C_{\|}}{H} V\left[\alpha_{a}(a, b, H)+\alpha_{b}(a, b, H)\right]\right\}=\varepsilon_{\|} \\
\varepsilon_{x y} & =\varepsilon_{y x}=0 \\
\varepsilon_{z z} & =\varepsilon_{m}\left[1-\frac{N_{S} C_{\|}}{H} V \alpha_{H}(a, b, H)\right]^{-1}=\varepsilon_{\perp} .
\end{aligned}
$$

In order to calculate the $C$ factors in Eqs. (27) and (29), one has to express the pair correlation function $N_{s}(r)$. Rigorously, it accounts for the short-range and long-range ordering of the NPs and can be taken from the literature ${ }^{54}$ Nevertheless, since the calculations are based on dipole-dipole interactions, the most relevant parameter is the distance between neighbor NPs. The $C$ factors can thus be simplified by introducing the mean interparticle distance $\Lambda_{\|}$and assuming that the surface density $N_{s}(r)$ is equal to 0 for $r<\Lambda_{\|}$and is equal to $N_{s}$ for $r>\Lambda_{\|}$:

$$
\int_{r=0}^{\infty} N_{S}(r) \frac{d r}{r^{2}}=\int_{r=\Lambda_{\|}}^{\infty} N_{S} \frac{d r}{r^{2}}=\frac{N_{S}}{\Lambda_{\|}} .
$$

By writing explicitly the $C$ coefficients in Eq. (29) using Eq. (30), one gets

$$
\begin{aligned}
& \varepsilon_{y y}=\varepsilon_{x x}=\varepsilon_{m}\left(1+\frac{N_{S} \frac{V}{H}\left[\alpha_{a}(a, b, H)+\alpha_{b}(a, b, H)\right]}{2\left\{1-\frac{V}{8} \frac{N_{S}}{\Lambda_{\|}}\left[\alpha_{a}(a, b, H)+\alpha_{b}(a, b, H)\right]\right\}}\right)=\varepsilon_{\|} \\
& \varepsilon_{x y}=\varepsilon_{y x}=0 \\
& \varepsilon_{z z}=\varepsilon_{m}\left[1-\frac{N_{S} \alpha_{H}(a, b, H) \frac{V}{H}}{1+\frac{V}{2} \alpha_{H}(a, b, H) \frac{N_{S}}{\Lambda_{\|}}}\right]^{-1}=\varepsilon_{\perp} .
\end{aligned}
$$




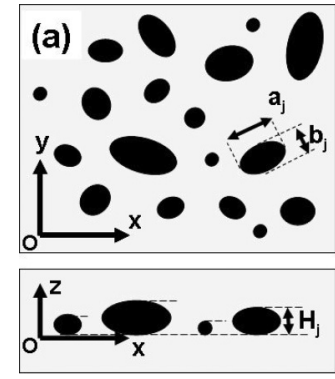

(b)

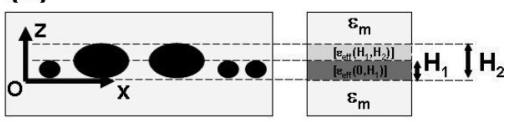

(c)

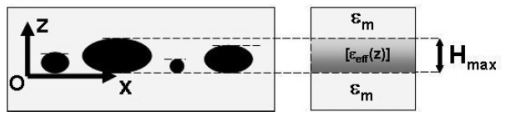

FIG. 2. (a) Schematic representation of a single layer of polydisperse NPs with correlated in-plane size, in-plane projected shape, and height distributions. (b) Effective medium corresponding to NPs with a bidisperse height distribution. (c) Effective medium corresponding to a continuous height distribution.

After a few maths, the expression of $\varepsilon_{\|}$in Eq. (31) yields a surface susceptibility equivalent to that obtained in Ref. 47. Let us moreover note that, if we assume a monodisperse system of spheroidal NPs ( $a=b$ and thus $\alpha_{a}=\alpha_{b}$ ), Eq. (31) leads to the Yamaguchi formulas ${ }^{36,38}$ written to match the case of NPs embedded in a homogeneous dielectric medium ${ }^{51,52}$ (i.e., with no image dipole field contributions) and with an isotropic in-plane organization. ${ }^{52}$

\section{EXTENSION: RANDOM IN-PLANE ORIENTATION AND ISOTROPIC IN-PLANE ORGANIZATION OF NPS WITH POLYDISPERSE HEIGHT DISTRIBUTION}

It has been shown ${ }^{48,49}$ that coalesced NPs embedded in a dielectric medium, grown by vapor deposition, present a polydisperse height distribution correlated to their in-plane size and inplane shape distributions [see Fig. 2(a)]. In order to achieve a realistic modeling of the optical response of such polydisperse systems of NPs, one thus has to take into account the height distribution. For this purpose, we develop here an approach based on a graded effective medium modeling, in the case of a ran- dom in-plane orientation and isotropic in-plane organization of the NPs. As a typical example, let us first consider a single layer of NPs with a bidisperse height distribution [as shown in Fig. 2(b)], the NPs of the first class (respectively, second class) presenting in-plane axes lengths $a_{1}$ and $b_{1}$ (respectively, $a_{2}$ and $b_{2}$ ) and a height $H_{1}$ (respectively, $H_{2}$ ). If taken separately, the single layer of NPs of the first class (respectively, second class) could thus be modeled as an effective layer of thickness $t_{1}=H_{1}$ (respectively, $t_{2}=H_{2}>H_{1}$ ) with the corresponding in-plane dielectric functions being derived from Eq. (29):

$$
\begin{aligned}
\varepsilon_{\|, 1} & =\varepsilon_{m}+\varepsilon_{m} \frac{N_{S, 1} C_{\|, 1}}{H_{1}} V_{1}\left[\alpha_{a}\left(a_{1}, b_{1}, H_{1}\right)+\alpha_{b}\left(a_{1}, b_{1}, H_{1}\right)\right] \\
\varepsilon_{\|, 2} & =\varepsilon_{m}+\varepsilon_{m} \frac{N_{S, 2} C_{\|, 2}}{H_{2}} V_{2}\left[\alpha_{a}\left(a_{2}, b_{2}, H_{2}\right)+\alpha_{b}\left(a_{2}, b_{2}, H_{2}\right)\right] .
\end{aligned}
$$

Under this form, the effective dielectric functions in Eq. (32) consist of a sum of two contributions: one from the dielectric medium, one from the NPs that tends toward zero when the NPs surface density decreases. The second term in both sums can thus be seen as an "excess quantity" that makes the effective dielectric function depart from that of the dielectric medium. Now, when considering the bidisperse single layer of NPs as a whole, one has to take into account the excess quantities related to both classes of NPs. More precisely, we will derive the effective response of the bidisperse single layer of NPs from a superposition of the excess quantities related to both classes of NPs. Following the assumptions made previously $\left(t_{1}=H_{1}\right.$ and $t_{2}=H_{2}$ ), we will sum the contributions of both classes of NPs in a bottom layer of thickness $H_{1}$ (i.e., for $0<z<H_{1}$ ), and only that of the second class will be considered in a top layer of thickness $H_{2}$ - $\mathrm{H}_{1}$ (i.e., for $H_{1}<z<H_{2}$ ), as depicted in Fig. 2(b).

Then, assuming that $C_{\|, 1}=C_{\|, 2}=C_{\|}$, i.e., that each NP interacts with NPs of both classes in an undifferentiated way, one obtains the in-plane effective dielectric functions in the two layers:

$$
\begin{gathered}
\varepsilon_{\|}\left(0, H_{1}\right)=\varepsilon_{m}+\varepsilon_{m} N_{S} C_{\|} \sum_{k=1,2} \frac{G_{k} V_{k}}{H_{k}}\left[\alpha_{a}\left(a_{k}, b_{k}, H_{k}\right)+\alpha_{b}\left(a_{k}, b_{k}, H_{k}\right)\right] \quad \text { for } \quad 0<z<H_{1} \\
\varepsilon_{\|}\left(H_{1}, H_{2}\right)=\varepsilon_{m}+\varepsilon_{m} N_{S} C_{\|} \frac{G_{2} V_{2}}{H_{2}}\left[\alpha_{a}\left(a_{2}, b_{2}, H_{2}\right)+\alpha_{b}\left(a_{2}, b_{2}, H_{2}\right)\right] \quad \text { for } \quad H_{1}<z<H_{2},
\end{gathered}
$$

where $N_{s}$ is the total surface density of NPs, and $G_{1}=N_{S, 1} / N_{S}\left(G_{2}=N_{S, 2} / N_{S}\right)$ is the fraction of NPs of the first (second) class. Let us note that, due to the different height of the NPs of the first and second classes, the corresponding dipoles are located in two distinct planes $\left(z=H_{1} / 2\right.$ and $\left.z=H_{2} / 2\right)$. In the conditions chosen for this paper, the distance between the two planes has an insignificant effect on the (weak) interaction between NPs, and can thus be neglected compared to the projected in-plane interparticle distance. One can then calculate the $C_{\|}$as in Sec. III B with $\Lambda_{\|}$being the projected in-plane interparticle distance. This approach can be generalized to an unlimited number $k_{\max }$ of classes $k$, assuming a correlation between the height $H_{k}$, the in-plane projected shape and the in-plane effective diameter $D_{k}$ of the NPs. The fraction, volume and polarizabilities of the NPs of the $k$ th class can thus be written $G\left(D_{k}\right), V\left(D_{k}\right), \alpha_{a}\left(D_{k}\right)$, and $\alpha_{b}\left(D_{k}\right)$, respectively, and one gets, in the thin layer verifying $H\left(D_{n}\right)<z<H\left(D_{n+1}\right)$ :

$$
\varepsilon_{\|}\left(H\left(D_{n}\right), H\left(D_{n+1}\right)\right)=\varepsilon_{m}\left\{1+N_{S} C_{\|} \sum_{k=n+1}^{k_{\max }} \frac{G\left(D_{k}\right) V\left(D_{k}\right)}{H\left(D_{k}\right)}\left[\alpha_{a}\left(D_{k}\right)+\alpha_{b}\left(D_{k}\right)\right]\right\} .
$$

Then, in the continuous limit, the value of the in-plane dielectric function for a given value of $z$ can be derived by replacing the sum over $k$ by an integral over the NPs in-plane diameter, the lower bound being the diameter $D(H=z)$ of NPs whose height 
is $z$. One thus obtains, after writing explicitly the $C$ coefficients:

$$
\varepsilon_{\|}(z)=\varepsilon_{m}\left[1+\frac{N_{S} \int_{D(H=z)}^{\infty} \frac{G(D) V(D)}{H(D)}\left[\alpha_{a}(D)+\alpha_{b}(D)\right] d D}{2\left(1-\frac{1}{8}\left[\int_{r=0}^{\infty} N_{S}(r) \frac{d r}{r^{2}}\right]\left\{\int_{D=0}^{\infty} G(D)\left[\alpha_{a}(D)+\alpha_{b}(D)\right] V(D) d D\right\}\right)}\right] .
$$

The dependence of $\varepsilon_{\|}$on $z$ is characteristic of a gradient of optical properties along the $z$ axis, as shown in Fig. 2(c) $z$ varying from 0 (bottom of the NPs) to $H_{\max }$ (maximum height of the NPs). Let us remark that, if a constant height is assumed for the NPs (as in Sec. III), the lower bound of the integral has to be set to 0 whatever $z$, thus leading to Eq. (28).

\section{SIMULATIONS}

\section{A. Monodisperse NPs}

\section{Effect of interaction between spherical NPs}

Monodisperse calculations have been performed using Eq. (31) for a single layer of silver NPs in a transparent dielectric medium. The dielectric function of the metal was taken from the Palik database and corrected for interface electron damping effects ${ }^{1}$, assuming the collision frequency $\Gamma$ of the electrons to depend, as a first approximation, on the effective in-plane NPs diameter D:

$$
\Gamma(D)=\Gamma_{0}+2 A v_{F} / D
$$

with $\Gamma_{0}$ and $\mathrm{v}_{\mathrm{F}}$ being the electron collision frequency and the Fermi velocity of the bulk metal, respectively. $A$ is the "damping factor" that we fix arbitrarily at 1 . The dielectric constant $\varepsilon_{m}$ of the dielectric medium was fixed arbitrarily at 4 . We focus here on the $\varepsilon_{\|}$component of the dielectric tensor, which describes the in-plane effective optical response of the material, as probed frequently by measurements at normal incidence. Simulations of the optical response at oblique incidence, which are beyond the scope of this paper, would require the calculation of both $\varepsilon_{\|}$and $\varepsilon_{\perp}$ dielectric functions. Figure 3(a) presents the spectrum of the in-plane extinction coefficient $k_{\|}$(imaginary part of the complex effective refractive index $\tilde{n}_{\|}$verifying $\left.\tilde{n}_{\|}^{2}=\varepsilon_{\|}\right)$of an assembly of spherical NPs $(a=b=H=D=15 \mathrm{~nm})$ with an interparticle (center-to-center) distance $\Lambda_{\|}=75 \mathrm{~nm}$. An absorption band peaking at around $500 \mathrm{~nm}$ can be seen, as a result of the in-plane SPR of the NPs, occurring upon excitation by an electric field oriented in the $x y$ plane.

The influence of the interparticle distance and thus of interaction between spherical NPs was probed by varying $\Lambda_{\|}$ between 20 and $75 \mathrm{~nm}, D$ being fixed at $15 \mathrm{~nm}$. As shown in Fig. 3(b), a decrease in $\Lambda_{\|}$below $40 \mathrm{~nm}$ induces a significant redshift of the SPR absorption band together with an increase in its width. Above $40 \mathrm{~nm}$, the spectral features of the SPR band are almost unaffected by $\Lambda_{\|}$, thus suggesting that interaction between NPs can be neglected for $L_{\|}>2.5 D$, as reported in other papers. ${ }^{55,56}$ In the following, $\Lambda_{\|}$will be fixed at $75 \mathrm{~nm}$ so that the effect of interaction between NPs can be neglected and the influence of the NPs shape and size distributions be studied independently of other effects.

\section{Effect of the NPs shape anisotropy}

Figure 4(a) shows the $k_{\|}$spectra of spherical NPs $(D=15 \mathrm{~nm}, b / a=1, H / D=1)$, flattened spheroidal NPs $(D=15 \mathrm{~nm}, b / a=1, H / D=0.66)$, and ellipsoidal NPs $\left[D=(a b)^{1 / 2}=15 \mathrm{~nm}, b / a=0.66, H / D=1\right]$. In the case of spherical and spheroidal NPs, the in-plane dielectric function of the metal was corrected for finite-size effects using Eq. (36), with $A=1$. For ellipsoidal NPs, shape anisotropy on the electron interface damping was taken into account by using the relations

$$
\Gamma(a)=\Gamma_{0}+2 A_{a} v_{F} / a \quad \text { and } \quad \Gamma(b)=\Gamma_{0}+2 A_{b} v_{F} / b
$$

along the long and short in-plane axes of the NP, respectively, as done in Refs. 51 and 57. We assume here that $A_{a}$ (longitudinal damping factor) and $\mathrm{A}_{b}$ (transverse damping factor) are equal to unity. As spherical NPs, flattened spheroidal NPs present one in-plane absorption band resulting from their in-plane SPR, which, however, peaks at a longer wavelength. In the case of ellipsoidal NPs, in-plane shape anisotropy $(a \neq b)$ splits the resonance into two modes corresponding to resonances along the short axis $b$ ("transverse mode") and long axis $a$ ("longitudinal mode") of the NPs. The evolution of the spectral position of these two modes is plotted in Fig. 4(b) as a function of the in-plane aspect ratio $b / a$ for different $\mathrm{H} / \mathrm{D}$ values $(D$ being fixed at $15 \mathrm{~nm})$. It can be seen that a decrease of $\mathrm{H} / \mathrm{D}$ at a given $b / a$ (flattening of the NPs) results in a comparable redshift of both resonance bands, at a rate increasing as $\mathrm{H} / \mathrm{D}$ decreases. Besides, the spectral shift
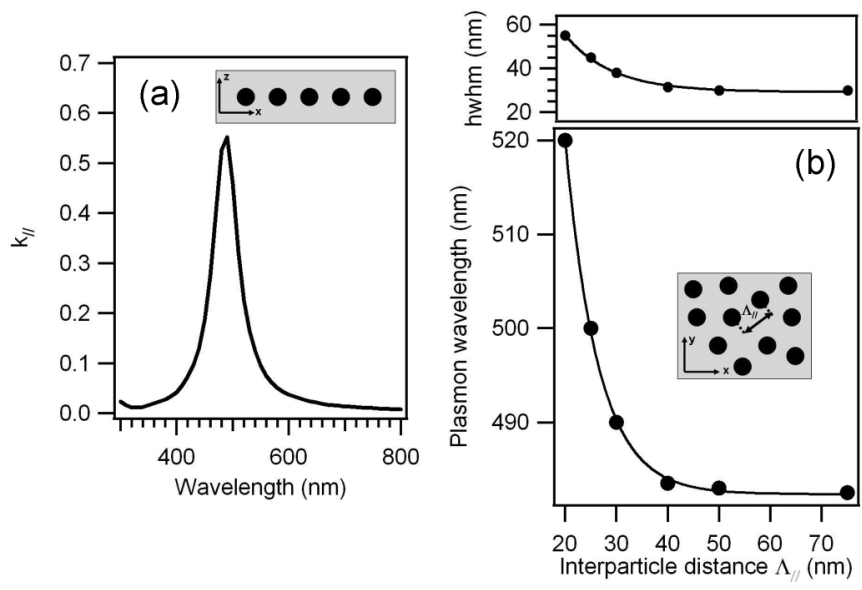

FIG. 3. (a) Calculated spectrum of the in-plane extinction coefficient $k_{\|}$of a single layer of monodisperse spherical Ag NPs (particle diameter $D=15 \mathrm{~nm}$, interparticle distance $\Lambda_{\|}=75 \mathrm{~nm}$ ) in a dielectric medium $\left(\varepsilon_{m}=4\right)$, (b) influence of $\Lambda_{\|}$on the spectral position and half-width at half-maximum (hwhm) of the SPR absorption band, $D$ being fixed at $15 \mathrm{~nm}$. 

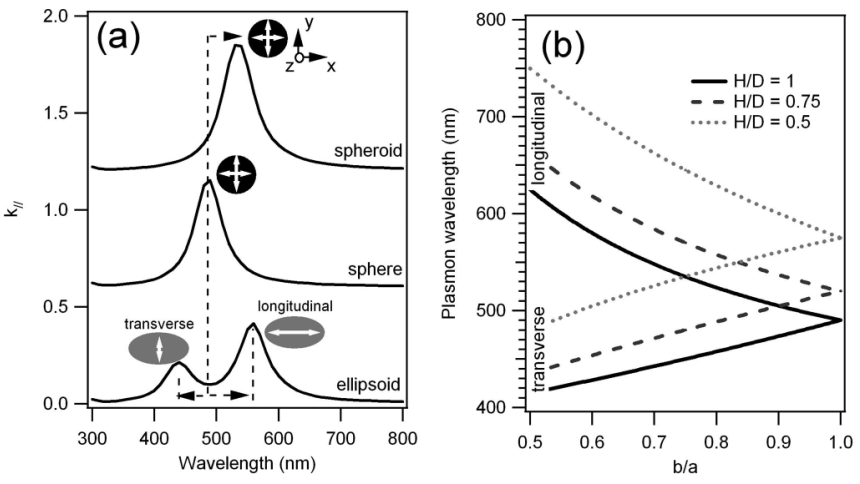

FIG. 4. (a) Calculated spectra of the effective in-plane extinction coefficient $k_{\|}$for a single layer of monodisperse spherical $(b / a=1$, $H / D=1)$, spheroidal $(b / a=1, H / D=0.66)$, and ellipsoidal $(b / a=$ $0.66, H / D=1) \mathrm{Ag}$ NPs in a transparent dielectric medium $\left(\varepsilon_{m}=4\right)$. (b) Evolution of the spectral position of the longitudinal and transverse SPR modes of the NPs as a function of $b / a$ and $H / D$. The distance $\Lambda_{\|}$between NPs was set at $75 \mathrm{~nm}$ and their in-plane effective diameter $D$ at $15 \mathrm{~nm}$.

between the two bands increases as $b / a$ decreases (in-plane elongation of the NPs).

\section{B. Polydisperse NPs with constant height}

\section{Spheroidal NPs with polydisperse in-plane diameter distribution}

The effect of the width of the in-plane diameter $D$ distribution on the optical response of a single layer of spheroidal NPs with constant height has been studied using Eq. (28), with $a=b$. Normalized Gaussian functions $G(D)$, shown in Fig. 5(a), were chosen to account for the $D$ distribution, with a half-width at half maximum (hwhm) $w$. The mean in-plane diameter $\langle D\rangle$ of the NPs was set at $15 \mathrm{~nm}$, their height $H$ at $11.25 \mathrm{~nm}$ [thus yielding the $H / D=11.25 / D$ relation, as shown in Fig. 5(a)]. The dielectric functions of the metal and the dielectric medium was the same as in Sec. V A, and
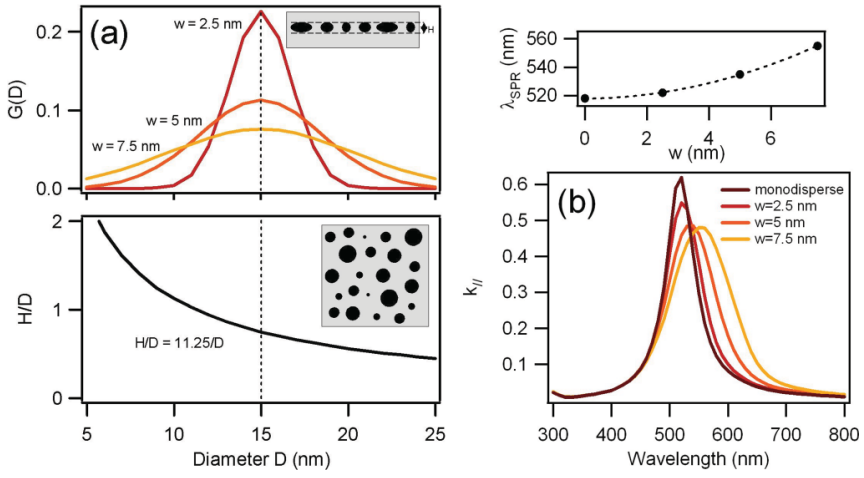

FIG. 5. (Color online) (a) Schematic representation of a single layer of spheroidal NPs, with a polydisperse distribution of in-plane diameter $D$ and a constant height $H=11.25 \mathrm{~nm}$ (thus yielding the correlation law $H / D(D)=11.25 / D)$, the Gaussian functions accounting for the $D$ distribution, and the $H / D(D)$ correlation law. (b) Calculated spectra of the in-plane extinction coefficient $k_{\|}$for several values of the hwhm $w$ of the $D$ distribution, and evolution of the spectral position of the SPR absorption band as a function of $w$. in-plane interface damping factors $A_{a}=A_{b}=1$ were taken for the metal using Eq. (37).

Figure 5(b) shows the $k_{\|}$spectra obtained for several values of $w$. Upon increasing $w$, it is observed that the SPR absorption band slightly shifts and broadens toward the red. At constant height, the larger the NPs diameter, the flatter their shape [as seen in Fig. 5(a)] and, according to Fig. 4(b), the longer the peak wavelength of their SPR absorption band. Thus the overall optical response of an assembly of NPs with polydisperse shape distribution results from contributions of absorption bands peaking at distinct wavelengths. Since the weight of the contribution of one spheroidal NP depends on its volume $V_{j}=\pi H_{j} D_{j}^{2} / 6$, which increases with $D_{j}$, the overall spectrum will be more influenced by the large and flat NPs of the distribution resonating at longer wavelengths than by the smaller ones. Thus the redshift and broadening of the SPR band observed in Fig. 5(b) are likely due to the broadening of the shape distribution and thus the strengthening of the contribution of the flattest and biggest NPs when $w$ increases. Let us moreover note that, due to the dependence of $V_{j}$ on $D_{j}{ }^{2}$, a broadening of the $D_{j}$ distribution induces an increase in the total volume occupied by the NPs. The total oscillator strength of the NPs assembly thus increases with $w$, in addition to the width of the shape distribution. Since a decrease in the amplitude of the SPR band is expected when the shape distribution broadens at a given total oscillator strength, the observed saturation in the peak amplitude (for $w>5 \mathrm{~nm}$ ) may result from the competition between the increase in the oscillator strength and the broadening of the shape distribution.

\section{Ellipsoidal NPs with correlated in-plane effective diameter and in-plane shape distributions}

The in-plane effective diameter distribution was taken into account, as previously, by normalized Gaussian functions. The correlation between the in-plane projected shape of the NPs and their in-plane effective diameter was described successively by two correlation laws of different slopes (typical of those found in the literature for NPs grown by vapor deposition ${ }^{48,49}$ ), which are displayed in Fig. 6(a). The mean in-plane diameter $\langle D\rangle$ of the NPs was set at $15 \mathrm{~nm}$ and the height $H$ at $11.25 \mathrm{~nm}$. In these conditions, the flatter, the bigger are the NPs. The dielectric function of the dielectric medium was the same as in the previous sections. The in-plane interface electron damping was taken into account using Eq. (37) with $A_{a}=A_{b}=1$. For a slowly decreasing $b / a$ (D) law [see Fig. 6(b)], it is seen that both SPR bands shift and broaden towards the red upon increasing $w$, these effects being more pronounced for the longitudinal mode than for the transverse mode. In the case of a rapidly decreasing $b / a$ (D) law [see Fig. 6(c)], a faster redshift and broadening of the longitudinal mode is observed, whereas the transverse mode slightly blue-shifts. As a result, the shift between the two modes increases upon increasing $w$ whatever the slope of the $b / a(D)$ law, but with higher values for a rapidly decreasing correlation law. As said previously in the case of polydisperse spheroidal NPs with a constant height, these trends can be related to the increasing contribution of large and flat NPs (low $H / D)$, which also present a strong in-plane elongation (low $b / a$ ). As discussed in Sec. V A2 [see Fig. 4(b)], 

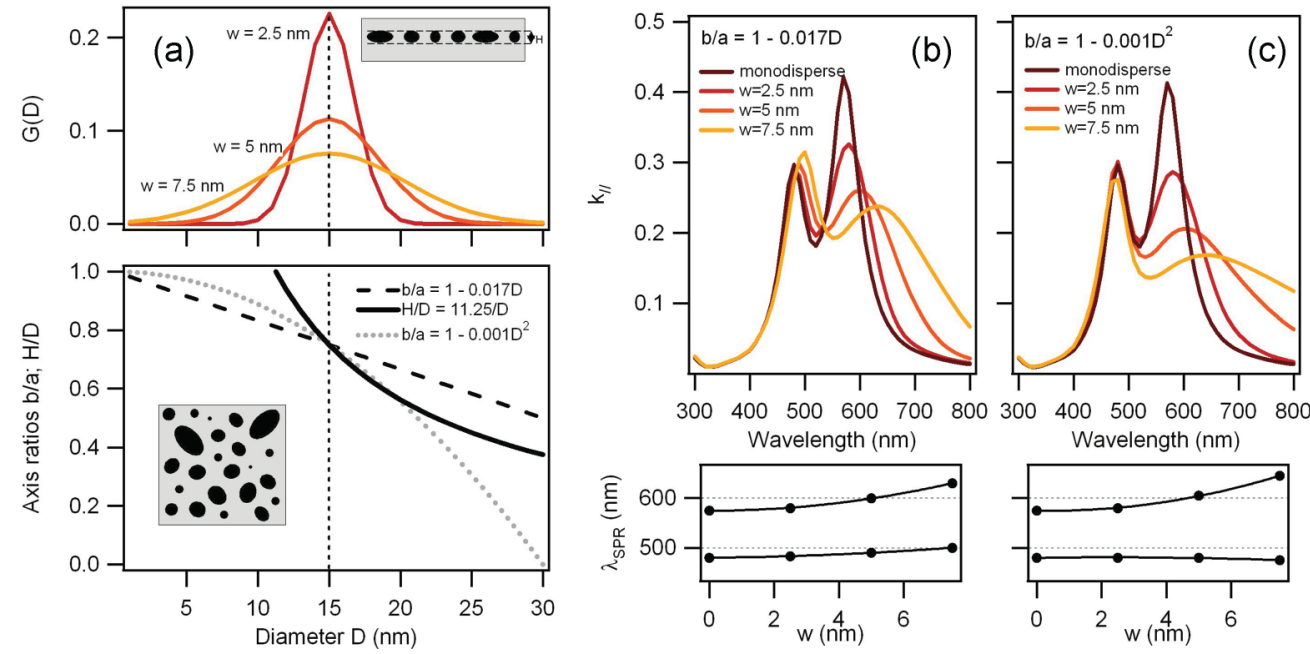

FIG. 6. (Color online) (a) Schematic representation of a single layer of ellipsoidal NPs, with a polydisperse distribution of in-plane diameter $D$ and a constant height $H=11.25 \mathrm{~nm}$ (thus yielding the relation $H / D=11.25 / D$ ). Gaussian functions accounting for the $D$ distribution, $b / a(D)$ and $H / D(D)$ correlation laws used for the calculations. Calculated spectra of the in-plane extinction coefficient $k_{\|}$as a function of the hwhm $w$ of the $D$ distribution: (b) for the slowly decreasing $b / a(D)$ law, (c) for the fastly decreasing $b / a(D)$ law, and the corresponding peak wavelengths.

a decrease in $H / D$ leads to an equal redshift of both modes, while a decrease in $b / a$ induces a blue shift (redshift) of the transverse (longitudinal) mode. In the case of decreasing $b / a(D)$ and $H / D(D)$ functions, the longitudinal mode will therefore redshift upon increasing $w$. This shift is expected to go faster when the slope of the $b / a(D)$ law increases, as observed from comparison between Figs. 6(b) and 6(c). Meanwhile, due to the competitive effect of decreasing $b / a$ and $H / D$, the transverse mode may blue-shift, redshift or not shift at all, depending on the respective slopes of both laws. The slight redshift (blue shift) of the transverse mode observed in Fig. 6(b) [in Fig. 6(c)] is thus due to the faster (slower) decrease of the $H / D(D)$ law when compared to the $b / a(D)$ law.

\section{Polydisperse NPs with polydisperse height distribution}

The effect of a polydisperse height distribution in the single layer of NPs on its effective optical response is twofold: (i) the $H / D(D)$ correlation law departs from that of Sec. VB. (scaling as $1 / D$ ) and (ii) the NPs layer is proposed to be described as a vertically graded material, i.e., by an effective dielectric tensor depending on $z$, following Eq. (35). The influence of the $H / D(D)$ correlation law on the SPRs has been studied by computing the effective optical response of the NPs layer at $z=0$ for a constant $H / D$ on the one hand, and a fast decreasing correlation law $\left(H / D=1-0.001 D^{2}\right)$ on the other hand. A slowly decreasing $b / a(D)$ law was chosen $(b / a=1-0.017 D)$ and the electron damping was taken into account using Eq. (37) with $A_{a}=A_{b}=1$. The other structural parameters were the same as in the previous section $\left(\langle D\rangle=15 \mathrm{~nm}, \Lambda_{\|}=75 \mathrm{~nm}, \varepsilon_{m}=4\right)$. Figure 7 shows that, for a given hwhm $(w=2.5 \mathrm{~nm})$ of the Gaussian distribution of NPs in-plane diameter $D$, increasing the slope of the $H / D$ (D) distribution mainly leads to a decrease in the amplitude of the longitudinal SPR band, as was already observed upon increasing the slope of the $b / a(D)$ law (see Fig. 6). The ratio between the amplitudes of the transverse and longitudinal SPR thus depends on the $b / a(D)$ and $H / D(D)$ correlation laws, as a result of inhomogeneous broadening of the longitudinal SPR band. Let us note that the ratio between the amplitudes of these two bands can also be affected by interface electron damping. In case of anisotropic in-plane projected shape of the NPs $(a \neq b)$, one expects a stronger damping along their short axis, ${ }^{51,57}$ i.e., $A_{a}<A_{b}$. Upon increasing $A_{b}$ from 1 to 3 at fixed $A_{a}=1$, the transverse SPR band is gradually damped, as shown in Fig. 7, while the longitudinal band remains unchanged.

\section{TEST OF THE MODEL FOR REAL MATERIALS}

Model trilayers consisting of a single layer of Ag NPs sandwiched between two transparent dielectric layers were elaborated by means of ion beam alternate sputtering of $\mathrm{Ag}$ and nitride targets. Details about the fabrication procedure are
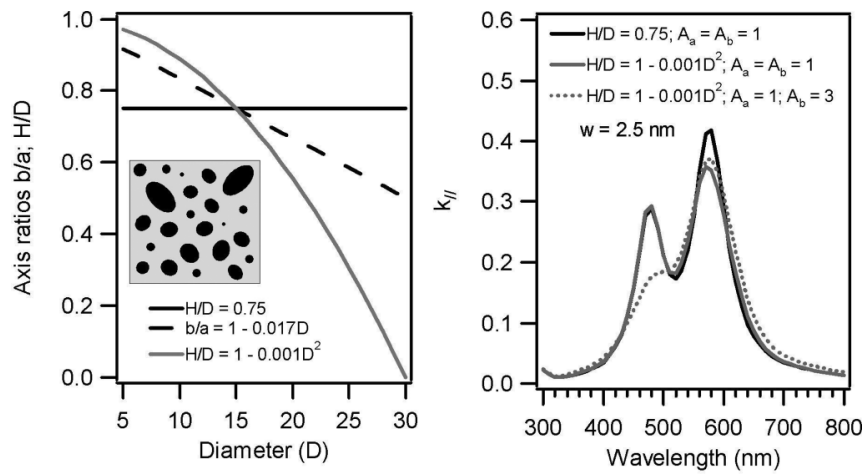

FIG. 7. Single layer of ellipsoidal NPs, with a polydisperse distribution of in-plane diameter $D$ and height $H$ : (left) $b / a(D)$ and $H / D(D)$ correlation laws used for the calculations, in which the hwhm $w$ of the $D$ distribution is fixed at $2.5 \mathrm{~nm}$ and $\langle D\rangle=15 \mathrm{~nm}$. (right) Spectra of the in-plane extinction coefficient $k_{\|}$obtained assuming a constant $H / D(H / D=0.75)$ and a fast decreasing $H / D$ (D) law $\left(H / D=1-0.001 D^{2}\right)$. The effect of varying the transverse damping coefficient $A_{b}$ is also studied. 

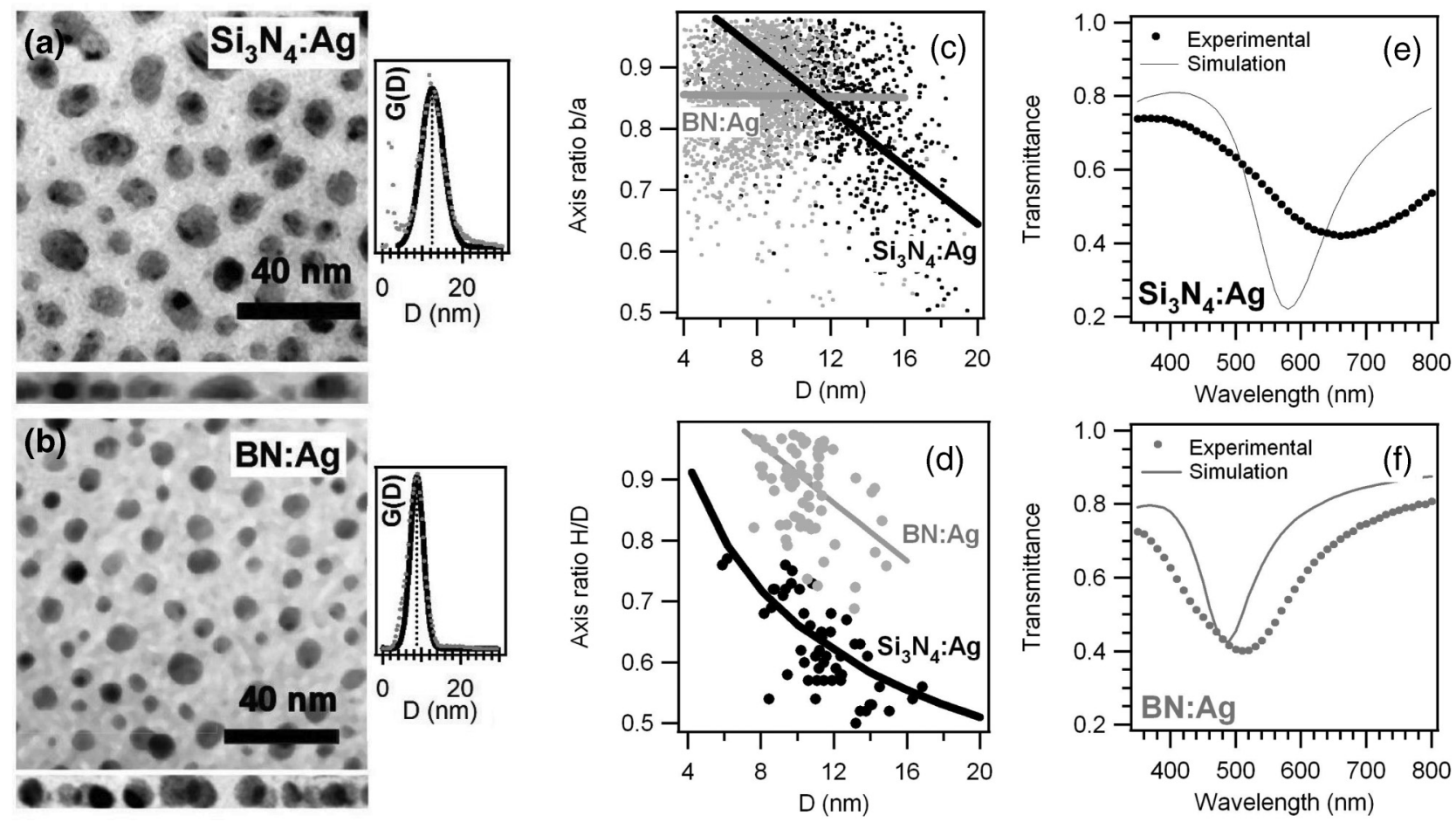

FIG. 8. Examples of TEM plane and cross-section views of the single layer of $\mathrm{NPs}$ in $\mathrm{Si}_{3} \mathrm{~N}_{4}: \mathrm{Ag}$ (a) and in $\mathrm{BN}$ : $\mathrm{Ag}$ (b) films, together with typical in-plane effective diameter $D$ histogram (dots) and the $G(D)$ Gaussian function used for the polydisperse calculations in Sec. VI (full line), (c) $b / a$ and (d) $H / D$ values of individual NPs (dots), and the $b / a(D)$ and $H / D(D)$ correlation laws considered for the two films (full lines). The transmittance spectra of the two films are also shown (e) and (f), together with simulated spectra obtained by using the Yamaguchi model (i.e., considering the NPs as monodisperse spheroids) and taking $A=1$ in Eq. (36). Grey dots and lines in (c), (d), (e), and (f) refer to the BN:Ag film, black dots and lines refer to the $\mathrm{Si}_{3} \mathrm{~N}_{4}: \mathrm{Ag}$ film.

given elsewhere. ${ }^{48}$ The nanostructure of the nanocomposite materials was studied by TEM using a JEOL 200CX electron microscope operated at $200 \mathrm{kV}^{48,51}$ Figure 8 shows examples of plane views and cross-section views of the single layer of NPs in dielectric/NPs/dielectric trilayers containing similar amounts of metal (with an effective metal thickness of about $2 \mathrm{~nm}$ ), one consisting of $\mathrm{Ag}$ NPs embedded in a $\mathrm{Si}_{3} \mathrm{~N}_{4}$ medium [ $\mathrm{Si}_{3} \mathrm{~N}_{4}:$ Ag film, Fig. 8(a)] and the other one of Ag NPs in a BN medium [BN:Ag film, Fig. 8(b)]. In both cases, the NPs can be approximated as ellipsoids with two of the principal axes in the layer's plane, as a result of coalescence events and partial reshaping during the Ag deposition. ${ }^{48}$ Moreover, it can be clearly seen that the NPs present polydisperse in-plane sizes, in-plane projected shapes, and heights. The size, shape anisotropy and related distributions clearly depend on the nature of the dielectric material: NPs present more anisotropic shapes when embedded in a $\mathrm{Si}_{3} \mathrm{~N}_{4}$ medium than in a $\mathrm{BN}$ medium. ${ }^{48}$ These trends were quantified by digital analysis of the TEM images, ${ }^{48,51}$ thus providing the average interparticle distance $\Lambda_{\|}$, the distributions of the in-plane effective diameter $D$, the in-plane axis ratio $b / a$ and the height-to-effective diameter ratio $H / D$ of the NPs. As shown in Figs. $8(\mathrm{c})$ and $8(\mathrm{~d})$, and as assumed in Sec. IV, the $H / D, b / a$, and $D$ distributions are correlated. Moreover, the $D$ distribution is broader for the NPs embedded in the $\mathrm{Si}_{3} \mathrm{~N}_{4}$ medium. These correlations can be approximated by bijective $b / a(D)$ and $H / D(D)$ laws, represented in Figs. 8(c) and 8(d) and explicited in Table I, together with the values of $\Lambda_{\|}$, and the average in-plane effective diameter $\langle D\rangle$ and hwhm $w$ of the $D$ distribution used in the following optical calculations for both films. Regarding the size of the NPs (around $10 \mathrm{~nm}$ ) and the interparticle distance (in the $15-20 \mathrm{~nm}$ range), it seems reasonable to calculate the optical response of the NPs assembly in the coupled dipole approximation ${ }^{22}$ on which this work is based.

The experimental transmittance spectra of both trilayers at normal incidence ${ }^{48}$ are presented in Figs. 8(e) and 8(f) (dots). They are dominated by one absorption band, peaking at $660 \mathrm{~nm}\left(\mathrm{Si}_{3} \mathrm{~N}_{4}: \mathrm{Ag}\right)$ and $510 \mathrm{~nm}(\mathrm{BN}: \mathrm{Ag})$, which is likely due to the longitudinal SPR of the NPs. In the case of the BN:Ag film, a shoulder located around $440 \mathrm{~nm}$ can be seen and could be due to the transverse SPR. Such a blue-shifted shoulder, although very weak, might also be present in the transmittance spectrum of the $\mathrm{Si}_{3} \mathrm{~N}_{4}: \mathrm{Ag}$ film. Rough simulations of the transmittance spectra (thin full

TABLE I. Structural parameters of both films as used in the optical calculations.

\begin{tabular}{lccccc}
\hline \hline Film & $\Lambda_{\|}(\mathrm{nm})$ & $\langle D\rangle(\mathrm{nm})$ & $w(\mathrm{~nm})$ & $b / a(D)$ & $H / D(D)$ \\
\hline $\mathrm{Si}_{3} \mathrm{~N}_{4}: \mathrm{Ag}$ & 18.5 & 12.5 & 3.9 & $1.12-0.024 D$ & $-0.038+1.58 D^{-0.35}$ \\
$\mathrm{BN}: \mathrm{Ag}$ & 14.6 & 8.8 & 2.3 & $0.86-0.0004 D$ & $1.15-0.024 D$ \\
\hline \hline
\end{tabular}


lines) performed using the Yamaguchi formulas simplified to match the case of NPs embedded in a homogeneous dielectric medium (i.e., considering the NPs as monodisperse spheroids $(w=0, b / a=1$, and $H / D=\langle H / D\rangle)$, with $\Lambda_{\|}$ and $\langle D\rangle$ taken from Table I) and taking $A=1$ in Eq. (36) yield a unique and too sharp absorption band peaking at a shorter wavelength than the measured dominant band. Taking into account the nonspheroidal shape of the NPs seems necessary to obtain a better agreement between simulation and experiment for the spectral position of the two SPR bands. Besides, reproducing accurately their shape, width, and amplitude requires taking the size/shape distributions into account and considering finite-size effects properly. The full set of structural data presented in Table I were thus used as input parameters for the calculation of the in-plane optical response of the single layer of embedded NPs in both cases (BN:Ag and $\mathrm{Si}_{3} \mathrm{~N}_{4}: \mathrm{Ag}$ ). For the sake of simplicity, the dielectric functions of $\mathrm{BN}$ and $\mathrm{Si}_{3} \mathrm{~N}_{4}$ were approximated as $\varepsilon_{\mathrm{BN}}=3.24$ and $\varepsilon_{\mathrm{Si3N} 4}=4$ (i.e., the higher order terms of the Cauchy law were neglected) for the effective medium calculations. We checked that such an approximation has no relevant effect on the effective response of the single layer of NPs. The dielectric function of a given NP was calculated using Eq. (37), assuming $A_{a}=\mathrm{A}_{b}=2$ for $\mathrm{Ag}$ NPs in BN and $A_{a}=4.5$ and $A_{b}=6$ for $\mathrm{Ag} \mathrm{NPs}$ in $\mathrm{Si}_{3} \mathrm{~N}_{4}$, these values being chosen so that simulated transmittance spectra could match reasonably the measured ones, as shown below. Such high values could reflect the existence of chemical damping events at the NPs/dielectric medium interface, as underlined in the works of Kreibig and coworkers. ${ }^{58}$ Since the NPs present a polydisperse height distribution, the NPs layer was described as a graded effective medium, using Eq. (35). For the sake of simplicity, discrete calculations were performed, in which the effective medium was considered as a stack of eight layers. The layer thicknesses were chosen to coincide with the height $H(D)$ of the NPs with $D=\langle D\rangle+/-0.5 n w$, $n$ being a nonzero integer. $H_{\max }$ was taken equal to 11.1 and $10.1 \mathrm{~nm}$ for the $\mathrm{BN}: \mathrm{Ag}$ and $\mathrm{Si}_{3} \mathrm{~N}_{4}: \mathrm{Ag}$ film, respectively. The obtained spectra of the in-plane extinction coefficient $k_{\|}$in these eight layers, numbered from 1 (bottom) to 8 (top) are shown in Fig. 9(a) (thin lines). Spectra calculated using Eq. (28) assuming a constant height $H=\langle H\rangle$ instead of the $H / D(D)$ correlation laws presented in Table I (thus considering the effective medium to be a homogeneous layer of thickness $t=\langle H\rangle$ ), are also shown for comparison (dashed lines). The calculated spectra of both $\mathrm{Si}_{3} \mathrm{~N}_{4}: \mathrm{Ag}$ and $\mathrm{BN}: \mathrm{Ag}$ films are dominated by the longitudinal SPR band (peaking around 600 and $500 \mathrm{~nm}$, respectively), the transverse mode appearing as a shoulder below $500 \mathrm{~nm}$. By plotting the evolution of the peak value of $k_{\|}\left(k_{\|, \max }\right)$ as a function of $z$ [see Fig. 9(b)], it is observed that the absorption of the NPs calculated from Eq. (35) (thin lines) remains constant in a layer whose thickness is similar to the NPs mean height $\langle H\rangle$, and then gradually decreases. In contrast, the calculations with a constant height $H=\langle H\rangle$ (dashed lines) yield a constant value for $k_{\|, \max }$ below $z=\langle H\rangle$ and a null value above. The direct consequence of this distinct behavior is that the absorption integrated over the whole thickness of the effective medium is higher when the effective medium is considered as a graded layer. Simulated transmittance spectra were computed from
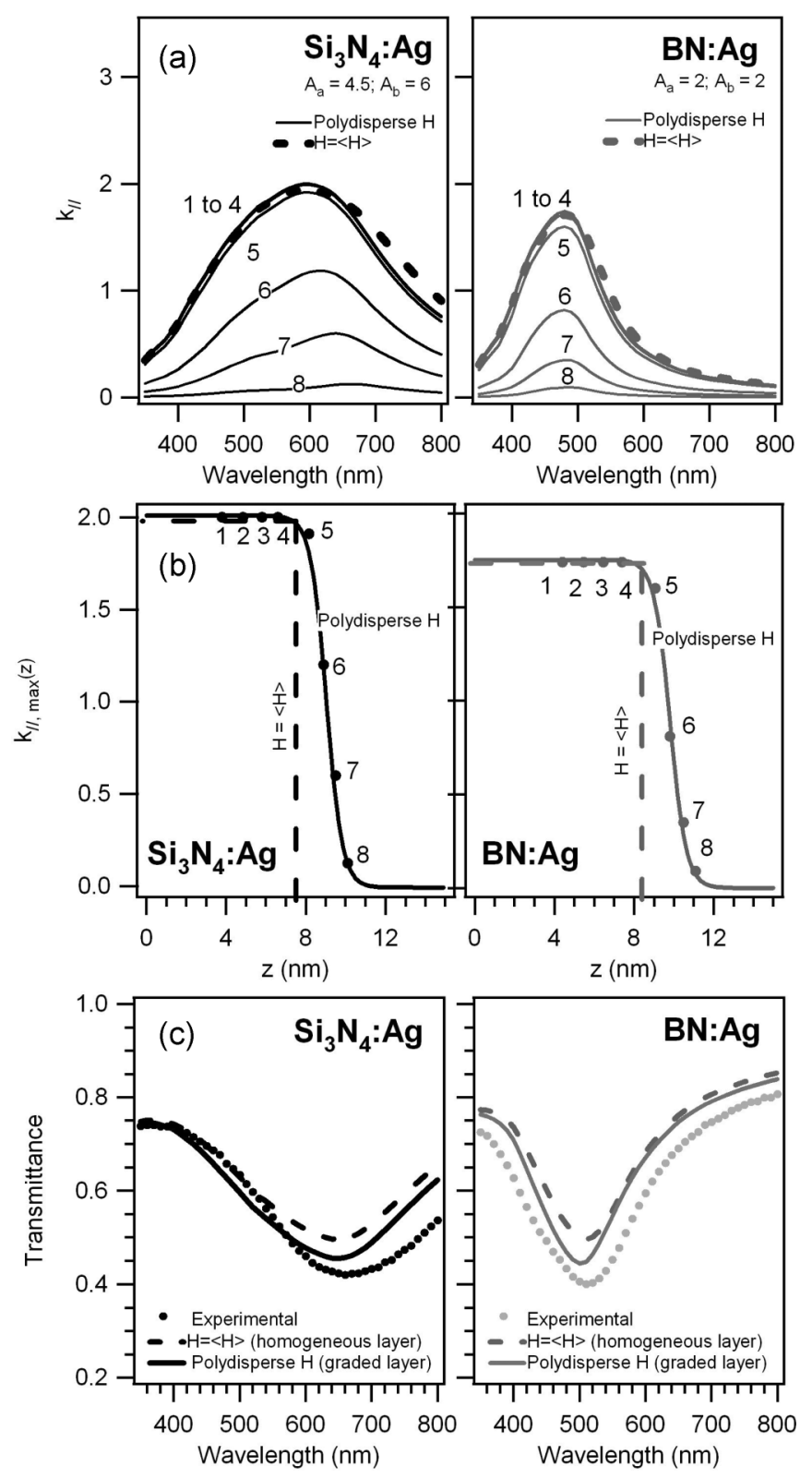

FIG. 9. (a) Spectra of the in-plane extinction coefficient $k_{\|}$ calculated in the eight layers of the graded effective medium accounting for the layer of NPs in the $\mathrm{Si}_{3} \mathrm{~N}_{4}$ : $\mathrm{Ag}$ (black) and the $\mathrm{BN}: \mathrm{Ag}$ (grey) films, using Eq. (35) (ellipsoidal NPs with polydisperse in-plane projected shape and height; full lines). For comparison, spectra calculated using Eq. (28) (ellipsoidal NPs with polydisperse in-plane projected shape and constant height $H=\langle H\rangle$; dashed lines) are presented. (b) Evolution of the peak value of $k_{\|}\left(k_{\|, \max }\right)$ as a function of $z$, for NPs with polydisperse height (full lines) and at constant height $H=\langle H\rangle$ (dashed lines). (c) Comparison between experimental (dots) and simulated transmittance spectra obtained from Eq. (28) (ellipsoidal NPs with a constant height and in-plane polydispersity, dashed lines) and from Eq. (35) (ellipsoidal NPs with in-plane polydispersity and height distribution, thin lines).

the $\varepsilon_{\|}$spectra obtained (i) using Eq. (28) assuming that all the NPs present the same height $\langle H\rangle$ [and are thus described by a homogeneous layer of thickness $t=\langle H\rangle$, dashed lines in Fig. 9(c)], (ii) using the $\varepsilon_{\|}(z)$ derived from Eq. (35) [i.e., considering the effective medium as a graded layer, thin lines 
in Fig. 9(c)]. The transmittance was calculated by multilayer modeling using the Abeles matrix formalism. The reflection and transmission of light at the interfaces, together with the propagation and absorption of light in each layer were taken into account in a coherent way. As shown in Fig. 9(c), the spectral position and shape of the measured longitudinal SPR band and transverse SPR shoulder (dots) can be reasonably reproduced if taking the ellipsoidal shape and polydispersity of the NPs into account together with finite size effects. Nevertheless, when assuming $H=\langle H\rangle$ (dashed lines), the transmittance is overestimated in the whole visible range. This overestimation, even if still present, is more moderate when the effective medium is considered as a graded layer (thin lines). The remaining discrepancy between simulated and measured spectra could be ascribed to the simplified description of the height distribution by Eq. (35). Indeed, the $H / D(D)$ [and $b / a$ (D)] functions depicted in Fig. 8 do not take into account the dispersion of $H / D$ (or $b / a$ ) values at a given $D$. The thickness of the graded effective layer and the slope of the gradient are thus likely underestimated by the model, leading to an underestimation of the absorption integrated over the whole thickness of the NPs layer. In addition, non-quasi-static effects such as dynamic depolarization ${ }^{24}$ and retarded dipole-dipole interaction, ${ }^{59}$ which are not taken into account by the model, can affect the amplitude of the SPR bands. Nevertheless, the influence of these two effects should be weak in the size, interparticle and wavelength ranges considered in this work. Experimental errors may also contribute significantly to the discrepancies between simulated and measured spectra. Indeed, TEM measurements are prone to an underestimation of the NPs size ${ }^{43}$ and thus to an underestimation of the NPs optical absorption by calculations based on TEM structural data.

\section{CONCLUSIONS}

We have presented a generalized effective medium model that permits to simulate the optical response of a single layer of polydisperse NPs embedded in a homogeneous dielectric medium, with the capability of taking into account the ellipsoidal shape of the NPs (with two of their principal axes in the layer's plane), and the (possibly correlated) distributions of their in-plane effective diameter $D$, their in-plane projected shape (through the $b / a$ aspect ratio) and height $H$. This model also takes into account the in-plane orientation and organization of the NPs. It can thus be derived to match, for instance, the case of an array of NPs oriented in a specific direction $^{11,60}$ or to that of NPs with an isotropic in-plane organization and a random orientation. In this paper, we have focused on the latter case, in order to test the accuracy of the model for a quantitative description of the optical response of single layers of polydisperse metal NPs embedded in a homogeneous dielectric medium, grown by vapor deposition in the coalescence regime. In such systems of NPs, correlation between the $D, b / a$, and $H$ distributions are usually evidenced. It has first been shown from simulations assuming a constant height $H$ for the NPs, that the distributions of $D$ and $b / a$, and the correlations between them significantly alter the symmetry, amplitude, width, and spectral position of the SPR absorption bands of the NPs. The calculations have then been extended to the case of a single layer of NPs with polydisperse height distribution, which has been tentatively described as a graded effective medium layer. Single layers of Ag NPs embedded in $\mathrm{BN}$ or $\mathrm{Si}_{3} \mathrm{~N}_{4}$ matrices finely characterized by TEM provide structural data that have been used for simulating the transmittance using the graded effective medium model. This approach yields an improved quantitative agreement between simulated and experimental spectra, which are, in contrast, poorly reproduced by the Yamaguchi model simplified to match the case of NPs embedded in a homogeneous dielectric medium. The obtained results suggest that a proper effective medium description should take into account the ellipsoidal shape, together with the size and shape distributions and the correlation between them. Moreover, a graded effective medium provides a slightly better empirical description of a single layer of NPs with polydisperse height distribution than a homogeneous effective layer. To our knowledge, these features have not been taken into account up to now in any other easily implementable model. Further work is, however, desired to take into account in a more accurate way the height distribution of the NPs, in order to evaluate the usefulness and correctness of the graded layer approach. Such works are of interest for the optical characterization of NPs of any kind (i.e., not only metal NPs): for instance, for semiconductor NPs, whose nondestructive characterization has been at the focus of recent studies. ${ }^{61,62}$

\section{ACKNOWLEDGMENTS}

The authors would like to thank Philippe Guérin and Marie-Françoise Denanot for the preparation and TEM characterizations of the nanocomposite films, respectively. J.T. acknowledges financial support from the Spanish Ministry of Science and Innovation (Grant No. JCI2009-05098 and project MAT2009-14369-C02-02).

\footnotetext{
*Corresponding author: johann.toudert@gmail.com

${ }^{1}$ U. Kreibig and M. Volmer, Optical Properties of Metal Clusters (Springer, Berlin, 1999).

${ }^{2}$ K. L. Kelly, E. Coronado, L. L. Zhao, and G. C. Schatz, J. Phys. Chem. B 107, 668 (2003).

${ }^{3}$ H. Ouacha, C. Hendrich, F. Hubenthal, and F. Träger, Appl. Phys. B 81, 663 (2005).

${ }^{4}$ S. Camelio, J. Toudert, D. Babonneau, and T. Girardeau, Appl. Phys. B 80, 89 (2005).

${ }^{5}$ J. Toudert, H. Fernandez, D. Babonneau, S. Camelio, T. Girardeau, and J. Solis, Nanotechnology 20, 475705 (2009).
}

${ }^{6}$ R. Del Coso, J. Requejo-Isidro, J. Solis, J. Gonzalo, and C. N. Afonso, J. Appl. Phys. 95, 2755 (2004).

${ }^{7}$ B. Lamprecht, A. Leitner, and F. R. Aussenegg, Appl. Phys. B 68, 419 (1999).

${ }^{8}$ J. R. Lakowicz, K. Ray, M. Chowdhury, H. Szmacinski, Y. Fu, J. Zhang, and K. Nowaczyk, The Analyst 133, 1308 (2008).

${ }^{9}$ P. Viste, J. Plain, R. Jaffiol, A. Vial, P. M. Adam, and P. Royer, ACS Nano 4, 759 (2010).

${ }^{10}$ H. A. Atwater and A. Polman, Nat. Mater. 9, 205 (2010).

${ }^{11}$ S. Camelio, D. Babonneau, D. Lantiat, L. Simonot, and F. Pailloux, Phys. Rev. B 80, 155434 (2009). 
${ }^{12}$ W. Rechsberger, A. Hohenau, A. Leitner, J. R. Krenn, B. Lamprecht, and F. R. Aussenegg, Opt. Commun. 220, 137 (2003).

${ }^{13}$ L. L. Zhao, K. L. Lance Kelly, and G. C. Schatz, J. Phys. Chem. B 107, 7373 (2003).

${ }^{14}$ H. Chen, X. Kou, Z. Yang, W. Ni, and J. Wang, Langmuir 24, 5233 (2008).

${ }^{15}$ T. W. H. Oates, H. Wormesteer, and H. Arwin, Prog. Surf. Sci. 86, 328 (2011).

${ }^{16}$ M. Losurdo, M. Bergmair, G. Bruno, D. Cattelan, C. Cobet, A. de Martino, K. Fleischer, Z. Dohcevic-Mitrovic, N. Esser, Melanie Galliet, R. Gajic, D. Hemzal, K. Hingerl, J. Humlicek, R. Ossikovski, Z. V. Popovic, and O. Saxl, J. Nanopart. Res. 11, 251 (2009).

${ }^{17}$ L. Simonot, D. Babonneau, S. Camelio, D. Lantiat, P. Guérin, B. Lamongie, and V. Antad, Thin Solid Films 518, 2637 (2010).

${ }^{18}$ R. Lazzari, G. Renaud, C. Revenant, J. Jupille, and Y. Borensztein, Phys. Rev. B 79, 125428 (2009).

${ }^{19}$ D. D. Evanoff Jr. and G. Chumanov, J. Phys. Chem. B 108, 13957 (2004).

${ }^{20}$ G. Mie, Ann. Phys. 3, 377 (1908).

${ }^{21}$ M. I. Mischenko, L. D. Travis, and D. W. Mackowski, J. Quantum Spectrosc. Radiat. Transfer 55, 535 (1996).

${ }^{22}$ B. Khlebtsov, A. Melnikov, V. Zharov, and N. Klebtsov, Nanotechnology 17, 1437 (2006).

${ }^{23}$ B. T. Draine and P. J. Flatau, J. Opt. Soc. Am. A 11, 1491 (1994).

${ }^{24}$ E. Hao and G. C. Schatz, J. Chem. Phys. 120, 357 (2004).

${ }^{25}$ T. Grosges, A. Vial, and D. Barchiesi, Opt. Express 13, 8483 (2005).

${ }^{26}$ J. Zhao, A. O. Pinchuk, J. M. McMahon, S. Li, L. K. Ausman, A. L. Atkinson, and G. C. Schatz, Acc. Chem. Res. 41, 1710 (2008).

${ }^{27}$ F. J. Garcia de Abajo and A. Howie, Phys. Rev. B 65, 115418 (2002).

${ }^{28}$ V. Myroschnychenko, J. Rodríguez-Fernández, I. Pastoriza-Santos, A. M. Funston, C. Novo, P. Mulvaney, L. M. Liz-Marzán, and F. J. Garcia de Abajo, Chem. Soc. Rev. 37, 1792 (2008).

${ }^{29}$ B. Palpant, B. Prével, J. Lermé, E. Cottancin, M. Pellarin, M. Treilleux, A. Perez, J. L. Vialle, and M. Broyer, Phys. Rev. B 57, 1963 (1998).

${ }^{30}$ J. Zuloaga, E. Prodan, and P. Nordlander, ACS Nano 4, 5269 (2010).

${ }^{31}$ D. J. Bergman, Phys. Rev. B 50, 3652 (1994).

${ }^{32}$ J. C. Maxwell-Garnett, Philos. Trans. R. Soc. London 203, 385 (1904).

${ }^{33}$ J. Toudert, D. Babonneau, S. Camelio, T. Girardeau, F. Yubero, J. P. Espinós, and A. R. Gonzalez-Elipe, J. Phys. D: Appl. Phys. 40, 4614 (2007).

${ }^{34}$ M. Gilliot, A. EnNaciri, L. Johann, J. P. Stoquert, J. J. Grob, and D. Muller, Phys. Rev. B 76, 045424 (2007).

${ }^{35}$ C. G. Granqvist and O. Hunderi, Phys. Rev. B 18, 2897 (1978).

${ }^{36}$ T. Yamaguchi, S. Yoshida, and A. Kinbara, Thin Solid Films 21, 173 (1974).
${ }^{37}$ S. Norrman, T. Andersson, C. G. Granqvist, and O. Hunderi, Phys. Rev. B 18, 674 (1978).

${ }^{38}$ V. A. Fedotov, V. I. Emel'yanov, K. F. MacDonald, and N. I. Zheludev, J. Opt. A: Pure Appl. Opt. 6, 155 (2004).

${ }^{39}$ M. Valamanesh, Y. Borensztein, C. Langlois, and E. Lacaze, J. Phys. Chem. C 115, 2914 (2011).

${ }^{40}$ D. Bedeaux and J. Vlieger, Physica 67, 55 (1973).

${ }^{41}$ J. Vlieger and D. Bedeaux, Thin Solid Films 69, 107 (1980).

${ }^{42}$ D. Bedeaux and J. Vlieger, Optical Properties of Surfaces (Imperial College Press, London, 2002).

${ }^{43}$ E. S. Kooij, H. Wormeester, E. A. M. Brouwer, E. Van Vroonhoven, A. Van Silfhout, and B. Poelsema, Langmuir 18, 4401 (2002).

${ }^{44}$ H. Wormeester, E. S. Kooij, A. Mewe, S. Rekveld, and B. Poelsema, Thin Solid Films 455, 323 (2004).

${ }^{45}$ J. M. Flores-Camacho, G. Weidlinger, N. Saucedo-Zeni, L. D. Sun, M. Hohage, and P. Zeppenfeld, Appl. Phys. A 98, 499 (2010).

${ }^{46}$ R. Lazzari, I. Simonsen, D. Bedeaux, J. Vlieger, and J. Jupille, Eur. Phys. J. B 24, 267 (2001).

${ }^{47}$ D. Bedeaux and J. Vlieger, Thin Solid Films 102, 265 (1983).

${ }^{48}$ J. Toudert, S. Camelio, D. Babonneau, M.-F. Denanot, T. Girardeau, J. P. Espinos, F. Yubero, and A. R. Gonzalez Elipe, J. Appl. Phys. 98, 114316 (2005).

${ }^{49}$ D. Lantiat, D. Babonneau, S. Camelio, F. Pailloux, and M. F. Denanot, J. Appl. Phys. 102, 113518 (2007).

${ }^{50}$ R. Lazzari and I. Simonsen, Thin Solid Films 419, 124 (2002).

${ }^{51}$ J. Toudert, D. Babonneau, L. Simonot, S. Camelio, and T. Girardeau, Nanotechnology 19, 125709 (2008).

${ }^{52}$ J. Toudert, L. Simonot, S. Camelio, and D. Babonneau, Appl. Opt. 49, 3630 (2010).

${ }^{53}$ C. F. Bohren and D. R. Huffman, Absorption and Scattering of Light by Small Metal Nanoparticles (Wiley, New York, 1988).

${ }^{54}$ P. W. Zhu and J. W. White, J. Chem. Phys. 104, 9169 (1996).

${ }^{55}$ S. Marhaba, G. Bachelier, C. Bonnet, M. Broyer, E. Cottancin, N. Grillet, J. Lermé, J. L. Vialle, and M. Pellarin, J. Phys. Chem. C 113, 4349 (2009).

${ }^{56}$ P. K. Jain and M. A. El Sayed, Chem. Phys. Lett. 487, 153 (2010).

${ }^{57}$ W. A. Kraus and G. C. Schatz, J. Chem. Phys. 79, 6130 (1983).

${ }^{58}$ U. Kreibig, G. Bour, A. Hilger, and M. Gartz, Phys. Status Solidi A 175, 351 (1999)

${ }^{59}$ C. G. Granqvist and O. Hunderi, Phys. Rev. B 16, 1353 (1977).

${ }^{60}$ J. R. Sánchez-Valencia, J. Toudert, A. Borras, A. Barranco, R. Lahoz, R. G. F. de la Fuente, F. Frutos, and A. R. Gonzalez-Elipe, Adv. Mater. 23, 848 (2011).

${ }^{61}$ R. J. Zhang, Y. M. Chen, W. J. Lu, Q. Y. Cai, Y. X. Zheng, and L. Y. Chen, Appl. Phys. Lett. 95, 161109 (2009).

${ }^{62}$ T. P. Chen, Y. Liu, M. S. Tse, O. K. Tan, P. F. Ho, and K. H. Liu, Phys. Rev. B 68, 153301 (2003). 